\title{
Analysis, fate and toxicity of chiral non-steroidal anti-inflammatory drugs in wastewaters and the environment: a review
}

\author{
Bruce Petrie $^{1}$ D $\cdot$ Dolores Camacho-Muñoz ${ }^{1}$
}

Received: 10 July 2020 / Accepted: 29 July 2020 / Published online: 20 August 2020

(c) The Author(s) 2020

\begin{abstract}
Non-steroidal anti-inflammatory drugs (NSAIDs) are found in the aquatic environment globally. Such drugs including naproxen, ibuprofen and ketoprofen are chiral molecules. Enantiomers of those drugs have identical physicochemical properties but can behave and interact differently in chiral environments due to differences in their three-dimensional shape. This results in enantiospecific differences in environmental fate and toxicity, which is often overlooked. Therefore, we review the analytical methods, occurrence and fate, and toxicity of chiral non-steroidal anti-inflammatory drugs at the enantiomeric level. The advancement of enantioselective chromatography methods, particularly the use of polysaccharide-based stationary phases, has enabled trace determination of enantiomers in complex environmental matrices. Macrocosm and microcosm studies of engineered and natural environments revealed that such drugs can undergo both enantioselective degradation and chiral inversion. Enantioselectivity has been reported during wastewater treatment, in surface waters and in agricultural soils. The use of microcosms spiked with individual enantiomers over racemates is essential to evaluate these degradation and inversion fate processes. The chiral inversion process whereby one enantiomer converts into its antipode can be significant if the more toxic enantiomers are formed. Existing enantiospecific effect studies report less than an order of magnitude difference in enantiomer toxicity. However, toxicity data for enantiomers are limited and further research is needed to better appreciate the environmental risk at the enantiomeric level.
\end{abstract}

Keywords Emerging contaminant $\cdot$ Enantiomeric fraction $\cdot$ Metabolite $\cdot$ River $\cdot$ Soil $\cdot$ LC-MS/MS

\section{Introduction}

The presence of pharmaceutical drugs in the environment has been an increasingly active area of research over the last 20 years. NSAIDs are a common therapeutic group of pharmaceuticals used to relieve pain and reduce inflammation. They act via inhibition of the cyclooxygenane isoenzymes (Brune and Patrignani 2015). NSAIDs are mainly excreted in urine as metabolites with a small proportion of the consumed dose being excreted unchanged. For example, $1 \%$ or less of naproxen, ibuprofen and ketoprofen is reported to be excreted unchanged in urine (Upton et al. 1980).

The extensive use of NSAIDs as prescription and overthe-counter medication results in the presence of $\mu \mathrm{g} \mathrm{\textrm {L } ^ { - 1 }}$ concentrations in untreated wastewater (Camacho-Muñoz

Bruce Petrie

b.r.petrie@rgu.ac.uk

1 School of Pharmacy and Life Sciences, Robert Gordon University, Aberdeen AB10 7GJ, UK et al. 2014; Cizmas et al. 2015; Gardner et al. 2013; Kasprzyk-Hordern et al. 2009; Larsson et al. 2014; Petrie et al. 2015; Roberts and Thomas 2006; Tijani et al. 2016). Furthermore, it is not uncommon for naproxen and ibuprofen concentrations to be greater than $10 \mu \mathrm{g} \mathrm{L}^{-1}$ in wastewater (Camacho-Muñoz et al. 2014; Kasprzyk-Hordern et al. 2009; Larsson et al. 2014; Petrie et al. 2015; Roberts and Thomas 2006). Incomplete removal during wastewater processing is reported for conventional wastewater treatment plants such as trickling filters and activated sludge (Camacho-Muñoz et al. 2012; Kasprzyk-Hordern et al. 2009; Petrie et al. 2015; Roberts and Thomas 2006; Verlicchi et al. 2012). Despite dilution of wastewater effluent upon discharge to surface waters, NSAIDs are found at $\mathrm{ng} \mathrm{L}^{-1}$ to $\mu \mathrm{g} \mathrm{L}^{-1}$ concentrations in the aquatic environment (Kasprzyk-Hordern et al. 2009; Petrie et al. 2015; Roberts and Thomas 2006).

NSAIDs are weakly acidic with acid-dissociation constants ( $p K a, \mathrm{pH}$ at which $50 \%$ of the drug is ionized) in the range 3.7-4.9 (Table 1). Therefore, at typical environmental $\mathrm{pH}$ values $(6.5-8.0)$ they will be ionized and negatively 


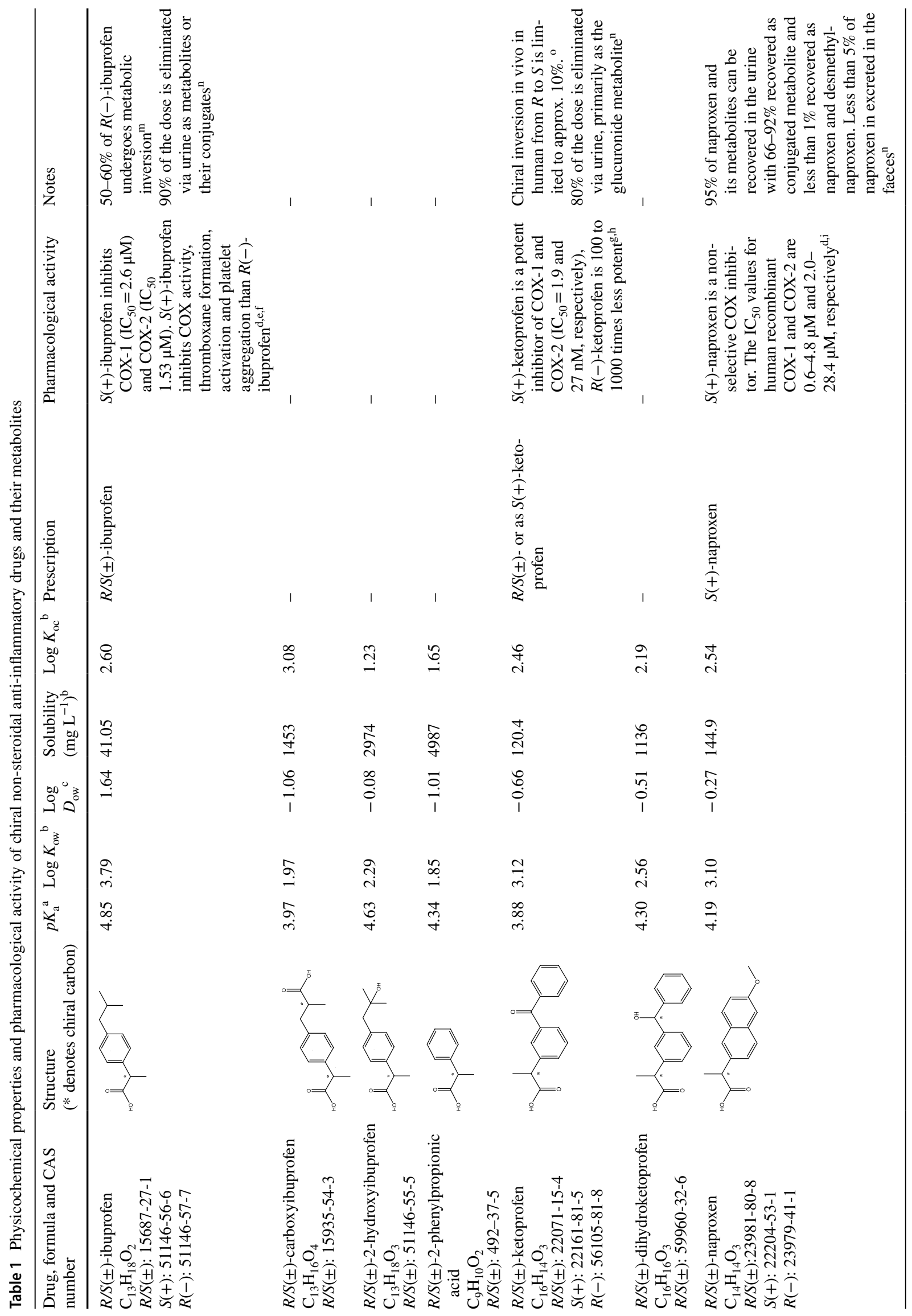




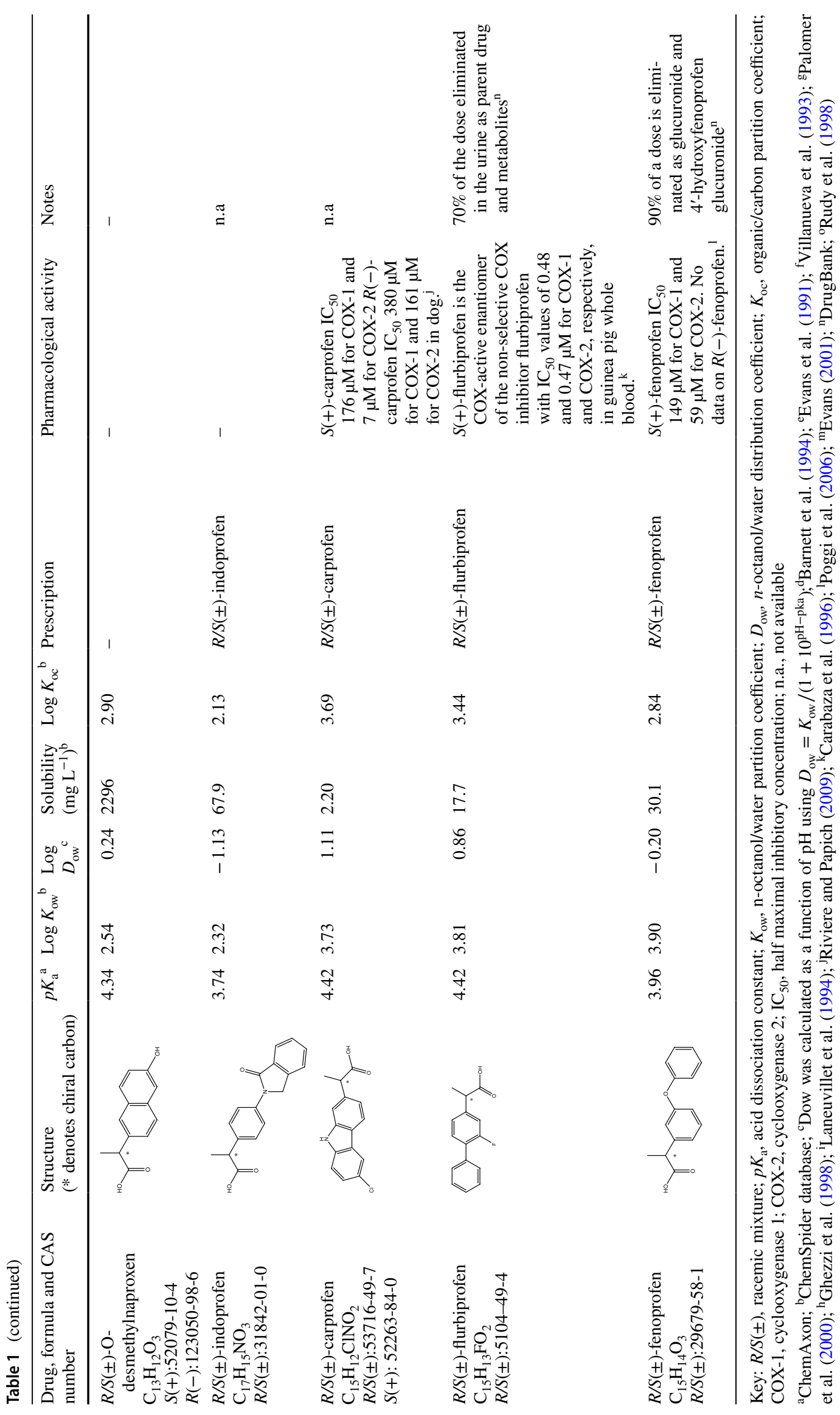


charged. Their $\mathrm{pH}$-dependent octanol-water partition coefficients $\left(\log D_{\mathrm{OW}}\right)$ range from -1.13 to 1.64 at $\mathrm{pH} 7$ (Table 1). Being charged enables electrostatic attractions with positively charged ions and surfaces in the environment. Other than the aqueous phase of environmental matrices, NSAIDs have been reported in the particulate phases of wastewater and activated sludge (Martín et al. 2012; Radjenović et al. $2009 \mathrm{~b}$ ) as well as being found in solid matrices including digested sludge, sediments and soils (Albero et al. 2014; Kumirska et al. 2015; Martín et al. 2012; Radjenović et al. 2009a).

Pharmaceutical drugs as a group of environmental pollutants remain termed emerging contaminants as their behaviour in the environment is not fully understood. Drug stereochemistry plays a significant role on the fate and effects of chiral pharmaceuticals in the environment (KasprzykHordern 2010; Sanganyado et al. 2017). However, NSAID stereochemistry in environmental settings is often overlooked. Therefore, the purpose of this review is to provide an up-to-date appraisal on the analysis, fate and toxicity of chiral NSAIDs, and to discuss recommendations for future research in this area.

\section{Chiral non-steroidal anti-inflammatory drugs}

Several NSAIDs are chiral including ibuprofen, naproxen, ketoprofen, indoprofen, flurbiprofen, carprofen and fenoprofen as well as several of their metabolites (Table 1). Enantiomers of chiral pharmaceuticals have identical chemical structures but different spatial arrangements of atoms around a stereogenic centre, e.g. see ketoprofen in Fig. 1a. Pairs of enantiomers have identical physicochemical properties but behave and interact differently in chiral environments due to differences in their three-dimensional shape. Consequently, chiral pharmaceuticals can exhibit enantioselectivity in environmental occurrence, fate and toxicity (Eaglesham et al. 2020; Kasprzyk-Hordern 2010; Ribeiro et al. 2012a, b, 2013; Sanganyado et al. 2017; Wong 2006). Pharmaceuticals with two stereogenic centres have two pairs of enantiomers and so on. Diastereomers are those stereoisomers that are not mirror images and can have different physicochemical properties, e.g. see the metabolite dihydroketoprofen in Fig. 1 b.

Differences that exist in the way chiral NSAIDs are dispensed. For example, ibuprofen is dispensed as the racemate,

(a)<smiles>C[C@@H](C(=O)O)c1cccc(C(=O)c2ccccc2)c1</smiles>

(S)-2-(3-benzoylphenyl)propanoic acid

(R)-2-(3-benzoylphenyl)propanoic acid

(b)

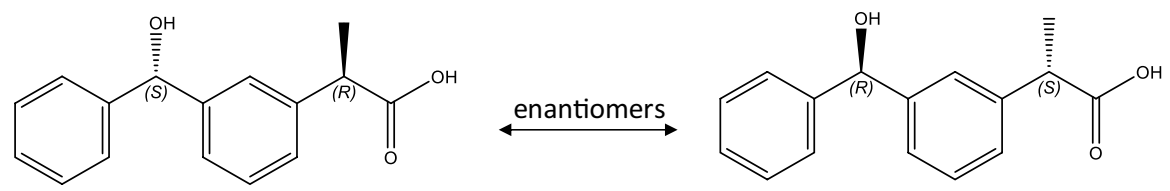

(R)-2-(3-((S)-hydroxy(phenyl)methyl) phenyl)propanoic acid

$$
\uparrow
$$

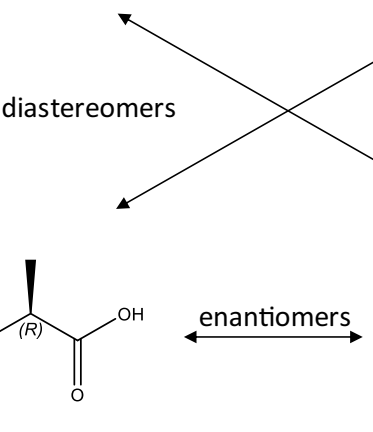

(R)-2-(3-((R)-hydroxy(phenyl)methyl) phenyl)propanoic acid
(S)-2-(3-((R)-hydroxy(phenyl)methyl) phenyl)propanoic acid<smiles>[3H][13CH3]</smiles><smiles>C[C@@H](C(=O)O)c1cccc([C@@H](O)c2ccccc2)c1</smiles>

(S)-2-(3-((S)-hydroxy(phenyl)methyl) phenyl)propanoic acid

Fig. 1 Enantiomers of ketoprofen (a) and enantiomers and diastereomers of dihydroketoprofen (b) 
i.e. equal dose of both enantiomers, and naproxen is dispensed in enantiopure form as $S(+)$-naproxen only. Naproxen is dispensed in this way because $R(-)$-naproxen causes hepatic toxicity, and the majority of the desired pharmacological activity resides with $S(+)$-naproxen (Harrington and Lodewijk 1997). Ketoprofen is dispensed as the racemate and as enantiomerically pure $S(+)$-ketoprofen (Table 1 ). As enantiomers of the same drug can behave differently in biologically mediated environments, enantioselective changes during human metabolism can occur.

Chiral NSAIDs are unlike many other chiral pharmaceuticals in that they can undergo chiral inversion whereby one enantiomer can convert into its antipode (Wsol et al. 2004). For example, unidirectional inversion of the biologically less active enantiomer $R(-)$-ibuprofen to the active enantiomer $S(+)$-ibuprofen has been reported in in vivo mammalian studies (Hao et al. 2005). The inversion process proceeds with an enzyme mediated reaction to form an activated Coenzyme A derivative of $R(-)$-ibuprofen (Fig. 2). This initial biotransformation step is considered to be enantioselective. The derivative is then racemized by an epimerase and cleaved by a hydrolase to release $S(+)$-ibuprofen (Kato et al. 2004, 2003). Racemization could result from the enzymatic deprotonation of the Coenzyme A derivative forming an enol-type intermediate (Khan et al. 2014). Enzymatic protonation can then take place either side of the planar double bond system.

Further enantioselectivity of chiral NSAIDs can also be observed during wastewater treatment and in the environment itself (Caballo et al. 2015a; Camacho-Muñoz et al. 2019; Hashim and Khan 2011; Kasprzyk-Hordern 2010; Khan et al. 2014; Matamoros et al. 2009; Sanganyado et al. 2017). This results in enantiomeric compositions in the environment being considerably different to that of the dispensed medication. Significantly, laboratory-based ecotoxicity testing of pharmaceuticals to assess environmental risk is typically undertaken using 'off-the-shelf' analytical reference standards. This can lead to the underestimation or overestimation of the associated environmental risk if enantiospecific toxicity exists. Nevertheless, an improved understanding on the importance of drug stereochemistry has resulted in more exposure and effect-driven studies of NSAIDs undertaken at the enantiomeric level (CamachoMuñoz et al. 2019; Yuan et al. 2018). This has been possible by the improvements in analytical methodologies capable of enantioselective determination at the $\mathrm{n} \mathrm{L}^{-1}$ concentrations found in the environment.

\section{Enantioselective analytical methods for non-steroidal anti-inflammatory drugs in environmental matrices}

The role of the analytical method is to achieve adequate separation of enantiomers such that their concentrations can be measured accurately. Traditionally, analytical methods capable of enantioselective analysis were used for quality control purposes in the manufacturing industry. Such methods facilitate the analysis of single drugs only, commonly making use of ultraviolet detection. These methods utilized chiral stationary phases for direct enantiomer separations using liquid chromatography (LC) which avoids the need for derivatization. These LC methods were typically operated in normal phase with mobile phases not compatible with mass spectrometry (MS). However, the use of MS is essential for environmental analysis due to the complexity of environmental matrices and the comparatively low concentrations of pharmaceutical drugs present (Kasprzyk-Hordern 2010; Ribeiro et al. 2012b, 2014; Sanganyado et al. 2017; Wong 2006). Therefore, new enantioselective methods have been developed to enable the coupling of the chromatographic system to MS specifically for environmental analysis. This
Fig. 2 Proposed mechanism of $R(-)$-ibuprofen inversion in rat liver. Adapted from Kato et al. (2003)<smiles>CC(C)Cc1ccc([C@@H](C)C(=O)O)cc1</smiles>

$R(-)$-Ibuprofen<smiles>CC(C)Cc1ccc([C@@H](C)C(=O)O)cc1</smiles>

S(+)-Ibuprofen

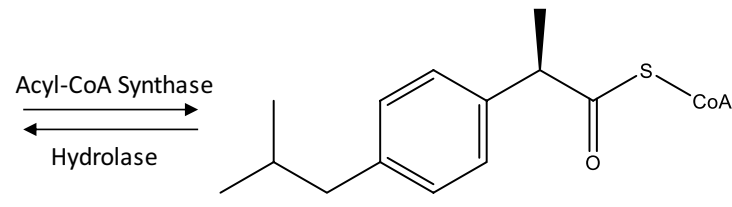

$R(-)-$ Ibuprofenyl-CoA

$\uparrow \mid \begin{gathered}\text { 2-Arylpropionyl-CoA } \\ \text { Epimerase }\end{gathered}$<smiles>CC(C)Cc1ccc([C@@H](C)C(=O)SC(C)(C)C)cc1</smiles>

S(+)-Ibuprofenyl-CoA 
also helps facilitate the simultaneous determination of several drugs in the same analytical run.

Enantioresolution $\left(R_{\mathrm{S}}\right)$ is a critical parameter to optimize during enantioselective method development. Ideally, $R_{\mathrm{S}}$ greater than 1.5 ensures baseline separation; however, $R_{\mathrm{S}}$ of 1.0 is considered adequate for quantitative purposes as it represents a maximum 2\% peak overlap (Bagnall et al. 2012). To date, enantioselective methodologies for NSAIDs have been developed for wastewater, river water, drinking water, sediments, sludge and fish tissues (Table 2). LC, gas chromatography (GC) and more recently, supercritical fluid chromatography (SFC) have been utilized as the separation method for environmental analysis. Prior to instrumental analysis, such methodologies require appropriate sample extraction and clean-up methods such as solid-phase extraction (SPE) and pressurized liquid extraction, depending on the matrix under investigation. Extraction methods are not considered enantioselective in nature and not discussed in this review. These have been reviewed extensively elsewhere, e.g. see (Boyac1 et al. 2015; Buchberger 2011; Evans and Kasprzyk-Hordern 2014; Farré et al. 2012; Llompart et al. 2019; Madikizela et al. 2018; Płotka-Wasylka et al. 2016; Zuloaga et al. 2012).

\section{Liquid chromatography-mass spectrometry}

Liquid chromatography (LC) is the most popular method of enantioselective analysis due to availability of chiral columns. Direct enantiomer separations typically rely on different stereospecific interactions with the chiral stationary phase. Chiral additives can also be added to the mobile phase to achieve direct separations; however, they are often not compatible with MS (Sanganyado et al. 2017). The direct approach relies on formation of transient diastereomeric complexes between the chiral selector and drug enantiomers in thermodynamic equilibria. The formation of these transient diastereomeric complexes for enantioseparation is driven by hydrogen bonds or ionic, ion-dipole, dipole-dipole, van der Waals and $\pi-\pi$ interactions (Scriba 2016). However, the precise mechanisms of enantioseparation remain poorly understood. Nevertheless, several tools are available to study the mechanism of chiral recognition including spectroscopic techniques, especially nuclear magnetic resonance spectroscopy. Molecular modelling is also proposed to visualize and analyze the dynamics of the process (Scriba 2016). Traditionally, the three-point interaction model was often used to describe the chiral recognition process (Bao et al. 2013; Pirkle and Pochapsky 1989). Here, three functional groups around the stereogenic centre of one of the enantiomers undergo molecular interactions with the chiral stationary phase (Berthod 2006). A variety of chiral stationary phases have been utilized for enantioseparation of NSAIDs including derivatized polysaccharides
(Camacho-Muñoz et al. 2016; Li et al. 2020; Ma et al. 2019; Wang et al. 2018; Yuan et al. 2018), glycopeptides (Camacho-Muñoz and Kasprzyk-Hordern 2017), glycoproteins (Camacho-Muñoz and Kasprzyk-Hordern 2015) and Pirkletype columns (Caballo et al. 2015a; Coelho et al. 2019).

\section{Chiral selector: polysaccharide phases}

The most popular phases for chiral NSAIDs are the derivatized polysaccharides (Table 2). Amylose tris-(3,5-dimethylphenylcarbamate), amylose tris-(3-chlorophenylcarbamate), amylose tris-(3-chloro-5-methylphenylcarbamate) and cellulose tris-(4-methylbenzoate) have all been used (Table 2). Such phases have a high number of chiral centres in the ordered polysaccharide backbone and substituents. Separation of carprofen, flurbiprofen, ibuprofen, indoprofen, ketoprofen, laxoprofen, naproxen and pranoprofen has all been achieved by polysaccharide phases (Table 2). Separation of NSAIDs is achieved in reversed phase mode under isocratic (Li et al. 2020; Ma et al. 2019; Yuan et al. 2018) and gradient elution mode (Camacho-Muñoz et al. 2016; Wang et al. 2018). Mobile phases typically consist of MS compatible, i.e. thermally labile, aqueous ammonium buffers such as formate or acetate, and organic modifiers such as acetonitrile or methanol. The type and concentration of buffer as well as the organic modifier influence enantioseparations (Camacho-Muñoz et al. 2016; Li et al. 2020; Ma et al. 2019; Wang et al. 2018; Yuan et al. 2018). For example, Li et al. (2020) reported a reduction in organic modifier of acetonitrile from 50 to $35 \%$ approximately doubled the flurbiprofen $R_{\mathrm{S}}$ on an amylose tris-(3-chlorophenylcarbamate) phase. Less significant changes to $R_{\mathrm{S}}$ were observed by varying the ammonium formate concentration from 20 to $30 \mathrm{mM}$ (Li et al. 2020).

Mobile phase $\mathrm{pH}$ also plays an important role in the separation. For example, when the analyte is fully ionized, it has a greater affinity with the aqueous mobile phase than the polysaccharide stationary phase. Polysaccharide phases such as amylose tris-(3,5-dimethylphenylcarbamate) do not have ionic sites within their structure for interaction with charged analytes (Ma et al. 2019). As the $p K a$ of the NSAIDs is 3.7-4.9 (Table 1), methods typically report the use of mobile phases with $\mathrm{pH}$ less than 5 (Table 2). However, care is needed as $\mathrm{pH}$ will also have a significant influence on MS sensitivity. Too low a $\mathrm{pH}$ will result in the neutral form of the drug predominating and poor MS response. Therefore, development of such methods for environmental analysis requires trade-offs between enantiomer separation and MS response. This could be overcome by post-column infusion of acidic/basic modifier which has been applied in LC-MS/ MS and SFC-MS/MS (Borges et al. 2011; Svan et al. 2015). Nevertheless, existing methods using polysaccharide-based phases (and appropriate sample preparation) report method detection limits (MDLs) at low $\mathrm{ng} \mathrm{L}^{-1}$ for aqueous matrices 


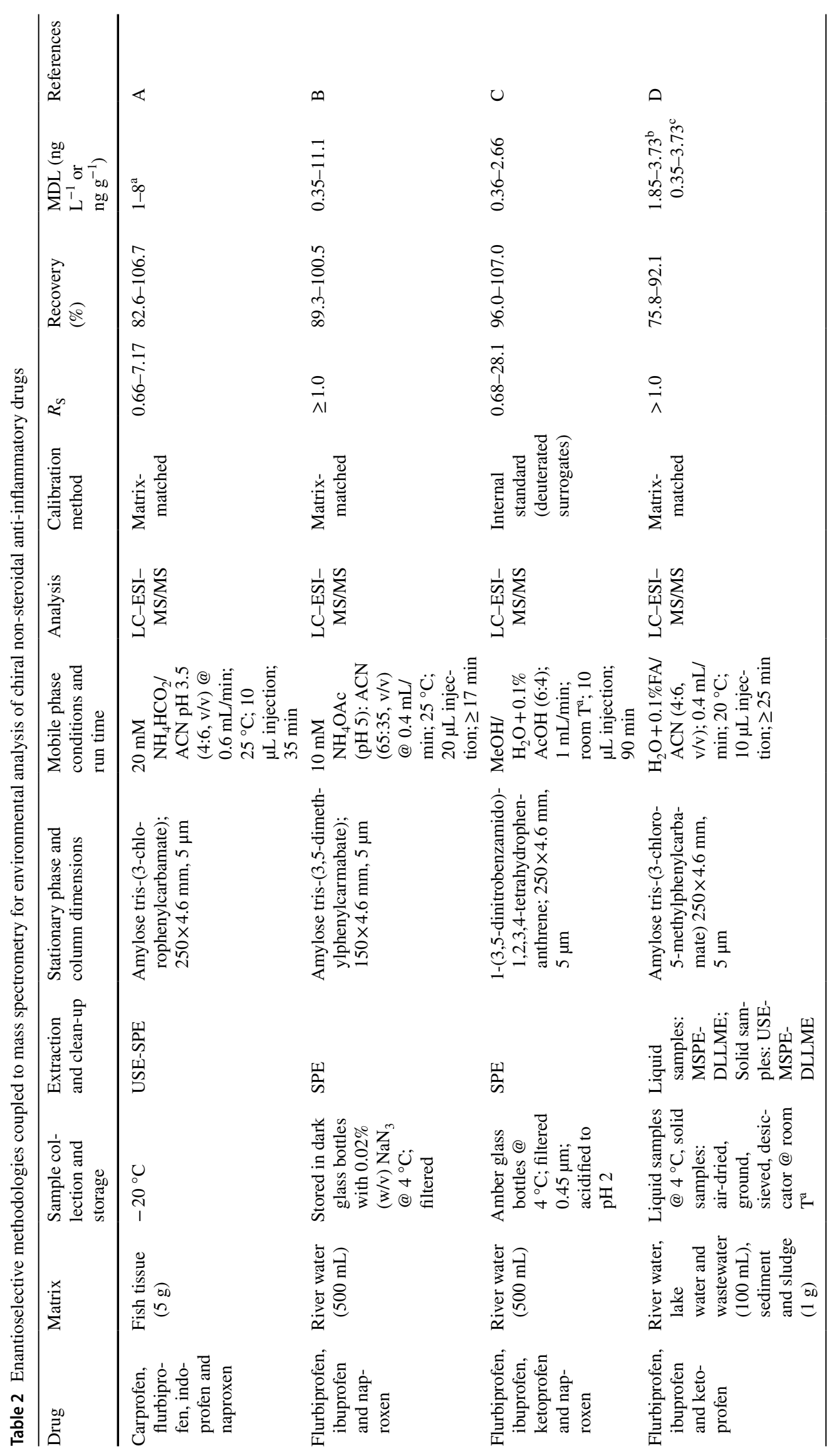




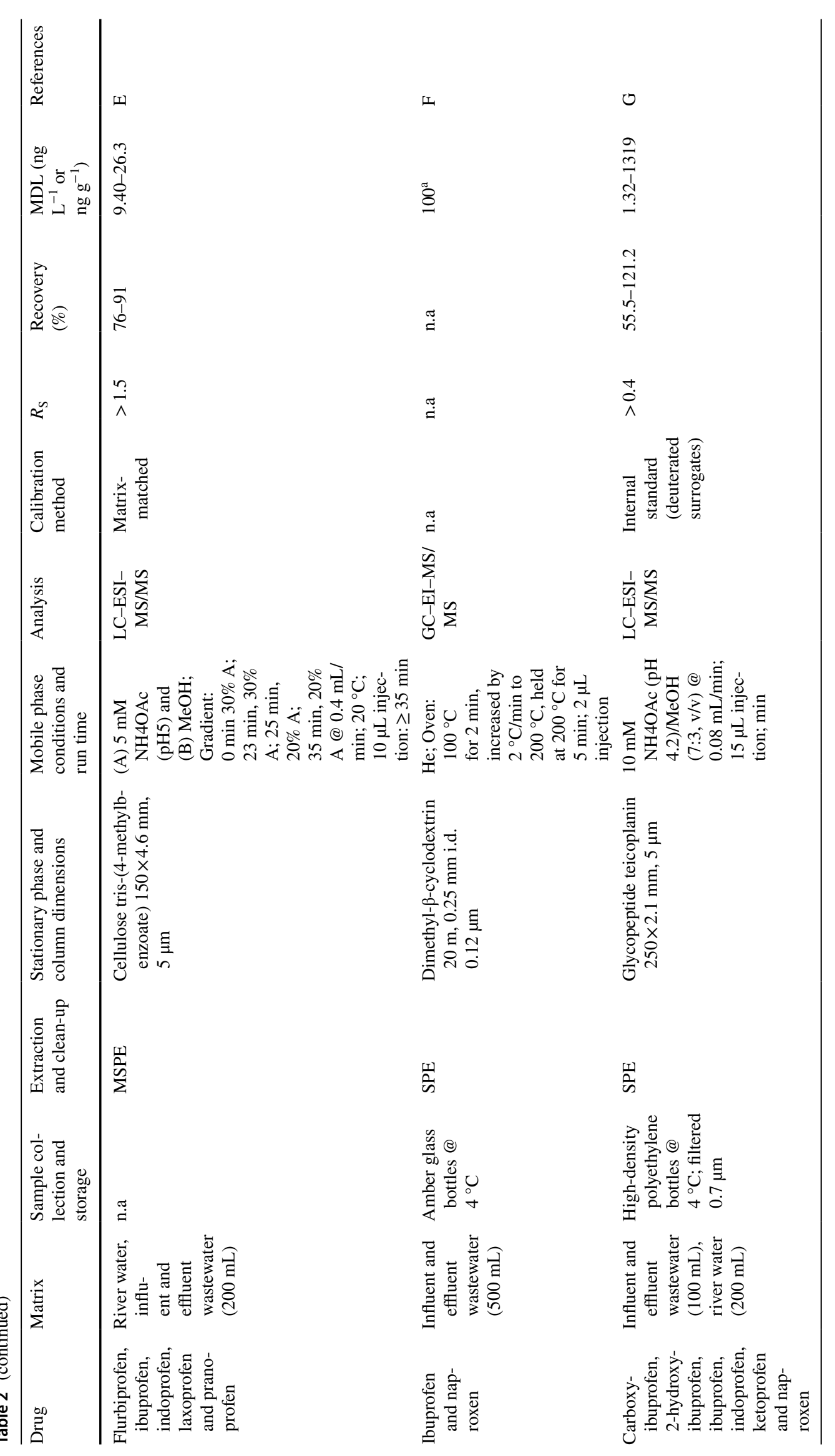




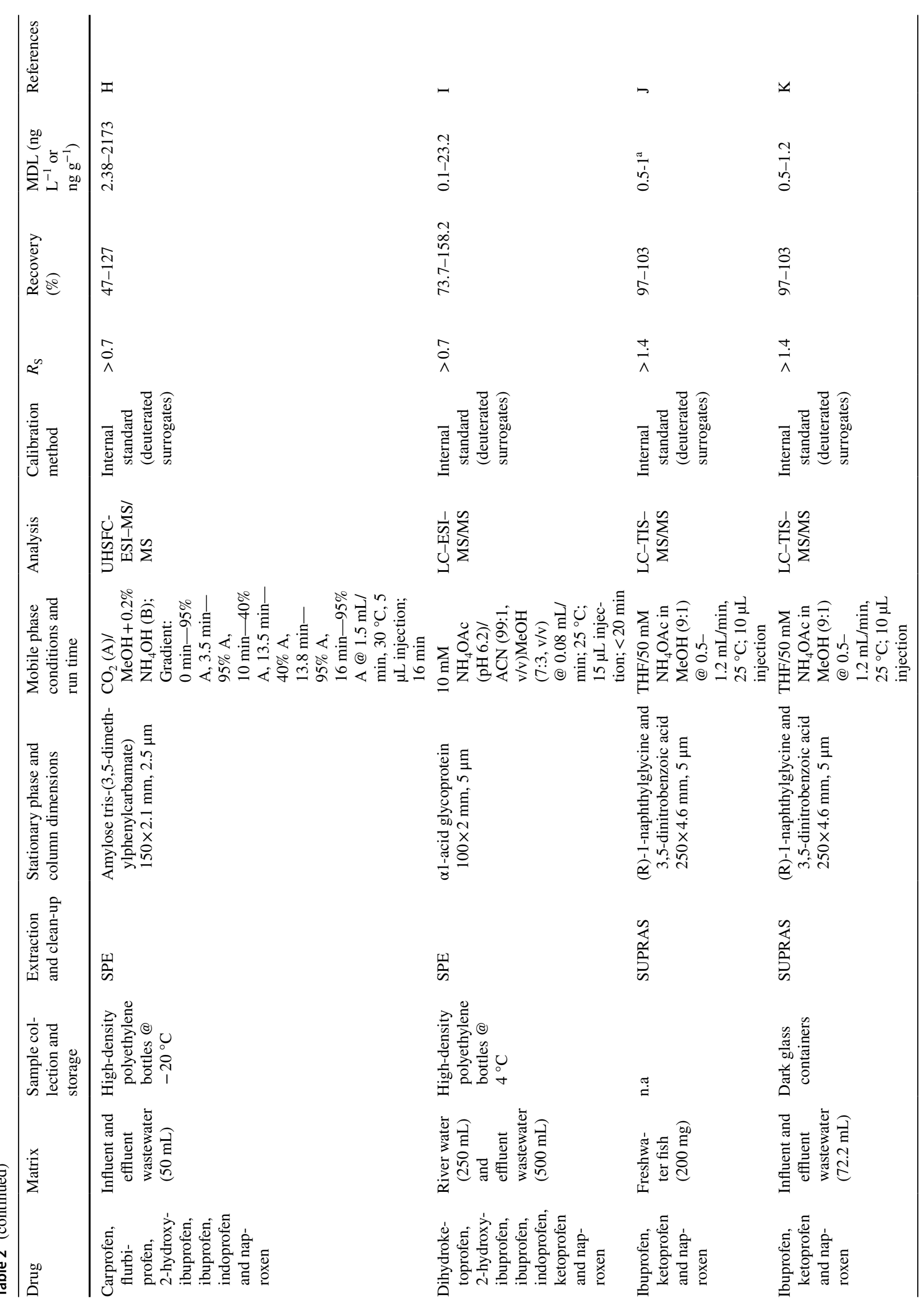




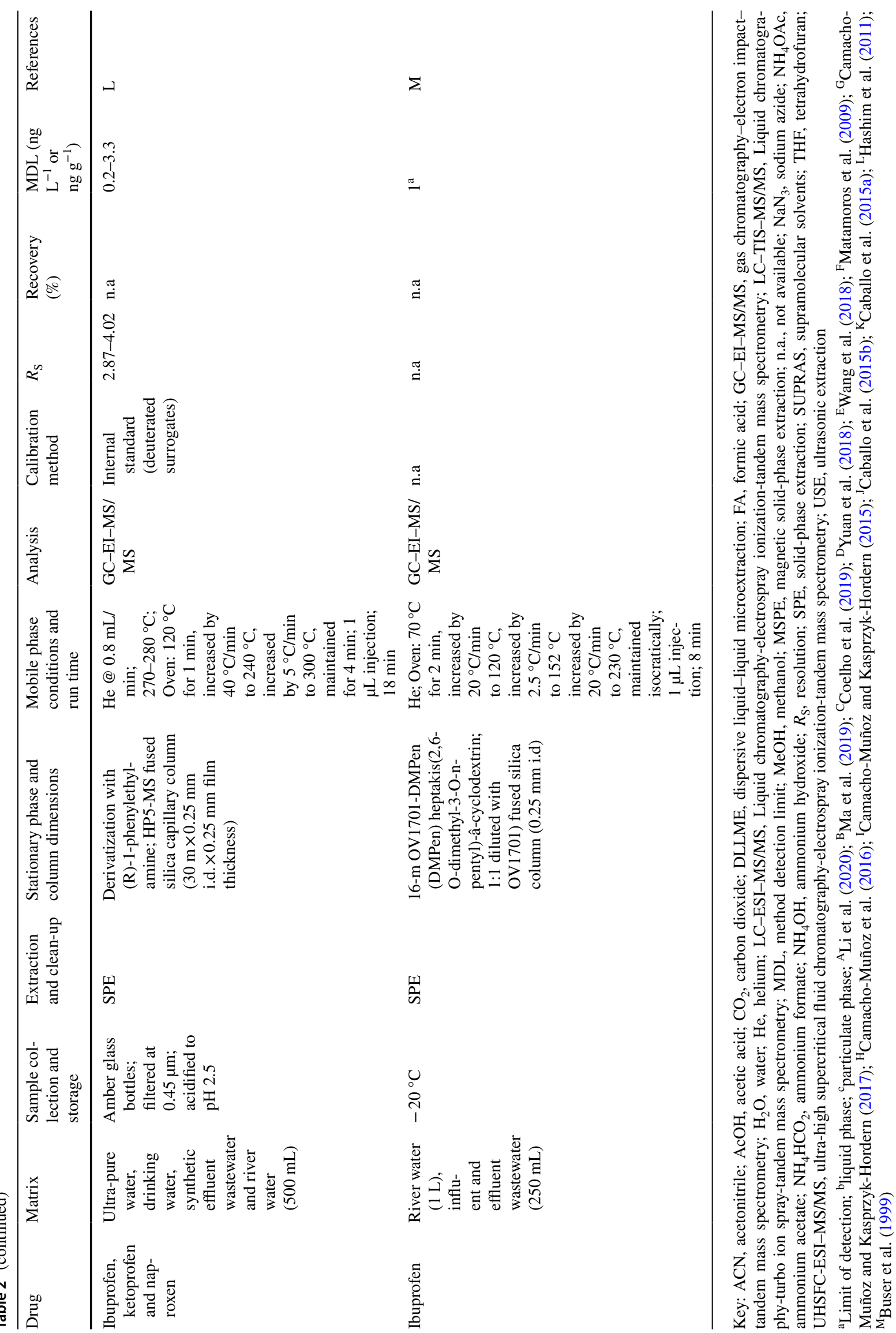


or $\mathrm{ng} \mathrm{g}^{-1}$ concentrations for solid matrices. Chromatographic run times range from 16 to $35 \mathrm{~min}$ (Table 2).

\section{Chiral selector: glycopeptide and glycoprotein phases}

Glycopeptide-based stationary phases have been widely applied for the separation of pharmaceutical drugs (Bagnall et al. 2012; Camacho-Muñoz and Kasprzyk-Hordern 2017; Evans et al. 2015; Petrie et al. 2018). Vancomycin is more suited to those basic drugs, e.g. beta-blockers, and teicoplanin for acidic drugs, e.g. NSAIDs. Teicoplanin has an isoelectric point of $\sim 3.5$, and electrostatic interactions are considered one of the dominant interactions occurring between the analytes and stationary phase. As the chiral selector itself is ionizable, changes to $\mathrm{pH}$ affects its degree of ionization as well as the analyte (Ilisz et al. 2013). Teicoplanin has been successfully applied for the simultaneous enantioseparation of indoprofen, naproxen, ketoprofen and ibuprofen as well as its metabolites carboxyibuprofen and 2-hydroxyibuprofen (Camacho-Muñoz and Kasprzyk-Hordern 2017). Separation was achieved in reversed phase mode with a mobile phase consisting of 70:30 (v/v) $10 \mathrm{mM}$ ammonium acetate at $\mathrm{pH}$ 4.2/methanol (Table 2). However, the chromatographic run time for the separation of all studied NSAIDs was $60 \mathrm{~min}$. A contributing factor to the comparatively long run time was the inclusion of additional drugs from different therapeutic groups including anthelmintic drugs, anti-cancer drugs, antibacterial drugs, central nervous system drugs and anti-fungal drugs within the same method.

The protein-based phase $\alpha_{1}$-acid glycoprotein (AGP) has also been successfully applied for the enantioseparation of NSAIDs (Table 2). This phase consists of a single peptide chain with 181 amino acids and five heteropolysaccharide units, containing 14 residues of sialic acid (Hermansson 1983). Due to the complexity of proteins, hydrophobic, ionic, $\pi-\pi$ and steric interactions, and hydrogen bonding are assumed to be the main retention mechanisms (Haginaka 2001). However, a limitation of AGP is the maximum content of organic modifier in the mobile phase cannot exceed $20 \%$. Furthermore, the operating $\mathrm{pH}$ range is limited to $\mathrm{pH} 4-7$. Nature and content of the organic modifier as well as the mobile phase $\mathrm{pH}$ are considered to have the greatest influence on enantioseparation using AGP phases (Hermansson and Hermansson 1994; Michishita et al. 2010). Successful separation has been achieved for ibuprofen, naproxen, ketoprofen and dihydroketoprofen using a mobile phase comprising 99:1 (v/v) $10 \mathrm{mM}$ ammonium acetate $(\mathrm{pH}$ 6.2):acetonitrile (Camacho-Muñoz and Kasprzyk-Hordern 2015). Separation of all NSAIDs was achieved in $20 \mathrm{~min}$ (Table 2).

\section{Chiral selector: Pirkle-type phases}

Pirkle-type stationary phases comprise a small chiral molecule bonded to a chromatographic support via a spacer (Fernandes et al. 2013; Pirkle and Pochapsky 1989). A number of different phases have been developed with the most popular being 1-(3,5-dinitrobenzamido)-1,2,3,4-tetrahydrophenanthrene. It was initially used for the separation of naproxen enantiomers (Welch 1994) and separates analytes with an aromatic system with a hydrogen-bond acceptor group near the stereogenic centre (Fernandes et al. 2013). Coelho et al. (2019) successfully developed a separation method for flurbiprofen, ibuprofen, ketoprofen and naproxen using a 1-(3,5-dinitrobenzamido)-1,2,3,4-tetrahydrophenanthrene stationary phase. The optimum mobile phase of 60:40 (v/v) methanol/0.1\% acetic acid required a chromatographic run time of $90 \mathrm{~min}$ (Table 2). On the other hand, Caballo et al. (2015a) used a Pirkle-brush column with a $(R)$-1-naphthylglycine and 3,5-dinitrobenzoic acid chiral selector for enantioseparation of ibuprofen, ketoprofen and naproxen. In this study, the mobile phase comprised 90:10 (v/v) tetrahydrofuran $/ 50 \mathrm{mM}$ ammonium acetate in methanol operated under a variable flow rate (Table 2). Under such conditions, the ammonium acetate concentration was reported to have the greatest influence on $R_{\mathrm{S}}$ using this stationary phase. The chromatographic run time was 36 min (Caballo et al. 2015a).

\section{Gas chromatography-mass spectrometry}

Chiral GC methods for the environmental analysis of NSAIDs and pharmaceuticals in general are limited (Buser et al. 1999; Hashim and Khan 2011). This is due to the nonvolatile nature of drugs requiring chemical derivatization to make them GC amenable. Hashim and Khan (2011) reported the derivatization of ibuprofen, ketoprofen and naproxen using $(R)$-1-phenylethylamine. This resulted in the conversion of the enantiomers to amide diastereomers facilitating indirect separation using a (5\%-phenyl)-methylpolysiloxane phase (Hashim and Khan 2011). The derivatization process was complete within $5 \mathrm{~min}$; however, an additional SPE treatment was required to separate the diastereomers from the derivatizing reagent. Nevertheless, chromatographic separation was achieved within $18 \mathrm{~min}$ and MDLs were in the range $0.2-3.3 \mathrm{ng} \mathrm{L}^{-1}$ and commensurate with those more sensitive LC methods (Table 1). An alternative methodology was utilized by Matamoros et al. (2009) whereby methylation of the carboxylic acid groups of ibuprofen and naproxen was performed using trimethylsulfonium hydroxide. Enantiomers were then separated directly on a chiral dimethyl-b-cyclodextrin column. In this case, the run time was $57 \mathrm{~min}$ (Table 2). 


\section{Supercritical fluid chromatography-mass spectrometry}

SFC-MS has also shown applicability for enantioselective analysis of pharmaceuticals (Camacho-Muñoz et al. 2016; Garzotti and Hamdan 2002; Hamman et al. 2011; Płotka et al. 2014). Supercritical fluids have the advantages of both gas states and liquid states (Płotka et al. 2014). SFC is normally operated similar to normal phase mode with carbon dioxide as the main mobile phase component enabling MS compatibility. Addition of organic modifiers to the mobile phase increases solvent strength. The viscosity and diffusivity of carbon dioxide make enantioselective separation possible in comparatively short run times, i.e. less than 10 min (Hamman et al. 2011; Płotka et al. 2014). However, the application of enantioselective SFC to environmental analysis is limited. Camacho-Muñoz et al. (2016) utilized an amylose tris-(3,5-dimethylphenylcarbamate) phase for the enantioseparation of carprofen, flurbiprofen, naproxen, ibuprofen and its metabolite 2-hydroxyibuprofen (Table 2). The mobile phase consisted of a gradient between carbon dioxide and $0.2 \%$ (v/v) ammonium hydroxide in methanol. Simultaneous enantioseparations were achieved within $16 \mathrm{~min}$.

\section{Occurrence and fate of non-steroidal anti-inflammatory drugs at the enantiomeric level in wastewater and the environment}

The development of robust analytical methodologies has facilitated the enantioselective study of chiral NSAIDs in complex environmental matrices. This includes wastewaters, receiving surface waters and soil systems where the occurrence and fate of NSAIDs have been investigated at the enantiomeric level.

\section{Wastewater}

\section{Influent wastewater}

Influent or untreated wastewater typically contains ibuprofen and naproxen at $\mu \mathrm{g} \mathrm{L}^{-1}$ concentrations and flurbiprofen and ketoprofen at sub- $\mu \mathrm{g} \mathrm{L}^{-1}$ concentrations (Camacho-Muñoz et al. 2014; Gardner et al. 2013; Kasprzyk-Hordern et al. 2009; Larsson et al. 2014; Petrie et al. 2015; Roberts and Thomas 2006). The enantiomeric composition of chiral drugs is typically reported as enantiomeric fraction (Sanganyado et al. 2020). The enantiomeric fraction of chiral NSAIDs can be calculated using Eq. (1):

Enantiomeric fraction $=\frac{S(+)}{[S(+)+R(-)]}$ where $S(+)$ is the $S(+)$-enantiomer and $R(-)$ is the $R(-)$ enantiomer. Therefore, a racemate, i.e. 50:50 mixture of two enantiomers, has an enantiomeric fraction of 0.5 , whereas an enantiomerically pure substance has an enantiomeric fraction of 0.0 or 1.0. There are several ways to calculate and report enantiomeric fraction (Tiritan et al. 2018). Therefore, to ensure consistency in presentation and data interpretation, all enantiomeric fraction data reported in this review are calculated using the above equation.

The ibuprofen enantiomeric fraction has been found to range from 0.63 to 0.94 ( $n=11$ studies) in Australia, China, Germany, Spain and UK influent wastewaters (Table 3). The enrichment of the pharmacologically active enantiomer $S(+)$-ibuprofen following its consumption as the racemate can be explained by the unidirectional inversion of $R(-)$ ibuprofen to $S(+)$-ibuprofen in the body (Buser et al. 1999). The consumption of enantiomerically pure $S(+)$-naproxen resulted in enantiomeric fractions equal to or greater than 0.88 ( $n=13$ studies) in influent wastewater (Table 3). Mammalian studies have shown $S(+)$-naproxen is not inverted into $R(-)$-naproxen (Sugawara et al. 1978; Wsol et al. 2004). The presence of comparatively low levels of $R(-)$-naproxen in influent wastewater could be a result of inversion of $S(+)$ naproxen during wastewater transport in the sewer network.

Wastewater typically takes between 0.5 and $24 \mathrm{~h}$ to arrive at the wastewater treatment plant. Sewer pipes have a biofilm that can result in drug biotransformation (Choi et al. 2020; Gao et al. 2019; Li et al. 2019). Naproxen and ibuprofen have been found to be stable in pilot scale gravity and rising main pipes over $8 \mathrm{~h}$ (Gao et al. 2019). However, enantioselective analysis was not undertaken to ascertain if inversion took place or not. Enantioselective changes of other drugs have been observed in influent wastewater previously (Castrignanò et al. 2017). However, further work is needed to ascertain the enantioselectivity of NSAIDs under sewer transport conditions. Ketoprofen has been reported in influent wastewaters with enantiomeric fractions ranging from 0.54 to 0.68 ( $n=5$ studies) in the UK, Spain and Australia (Table 3). The enrichment of $S(+)$-ketoprofen is likely to be a result of it being prescribed as both the racemate and enantiomerically pure $S(+)$-ketoprofen (Table 1$)$. Only about $10 \%$ of $R(-)$-ketoprofen is inverted to $S(+)$-ketoprofen in the body (Rudy et al. 1998). A single study reported the enantiomeric composition of flurbiprofen in influent wastewater with an enantiomeric fraction of 0.54 (Wang et al. 2018).

\section{Enantioselectivity during wastewater treatment and effluent wastewater composition}

Conventional biological wastewater treatment plants such as trickling filters and activated sludge are designed for carbonaceous material removal and can be adapted for nutrient removal. Removal of many pharmaceutical drugs is also 
Table 3 Reported occurrence of non-steroidal anti-inflammatory drugs at the enantiomeric level in environmental matrices

\begin{tabular}{|c|c|c|c|c|c|c|c|}
\hline Drug & Country & Analysis method & Sampled matrix & $R(-)$-enantiomer $\left(\mu \mathrm{g} \mathrm{L}^{-1}\right)$ & $S(+)$-enantiomer $\left(\mu \mathrm{g} \mathrm{L}^{-1}\right)$ & $\mathrm{EF}$ & References \\
\hline \multirow[t]{35}{*}{ Ibuprofen } & \multirow[t]{3}{*}{ UK } & \multirow[t]{3}{*}{ LC-MS/MS } & Influent wastewater ${ }^{\mathrm{TF}}$ & n.a & n.a & - & \multirow[t]{3}{*}{ A } \\
\hline & & & Effluent wastewater ${ }^{\mathrm{TF}}$ & 0.244 & 0.572 & 0.71 & \\
\hline & & & River water & $<0.009$ & $<0.009$ & - & \\
\hline & China & LC-MS/MS & River water & $<0.009-0.101$ & $<0.011-0.325$ & 0.70 & B \\
\hline & \multirow[t]{3}{*}{ China } & \multirow[t]{3}{*}{ LC-MS/MS } & Influent wastewater & 0.228 & 0.390 & 0.63 & \multirow[t]{3}{*}{$\mathrm{C}$} \\
\hline & & & Effluent wastewater & 0.036 & 0.075 & 0.68 & \\
\hline & & & River water & 0.040 & 0.075 & 0.62 & \\
\hline & \multirow[t]{2}{*}{ Europe } & \multirow[t]{2}{*}{ UHSFC-MS/MS } & Influent wastewater & $<1.41$ & 5.24 & $>0.79$ & \multirow[t]{2}{*}{$\mathrm{D}$} \\
\hline & & & Effluent wastewater & $<1.46$ & $<1.45$ & - & \\
\hline & \multirow[t]{2}{*}{ Spain } & \multirow[t]{2}{*}{ LC-MS/MS } & Influent wastewater ${ }^{\mathrm{AS}}$ & $0.256-0.415$ & $0.957-1.643$ & $0.79-0.86$ & \multirow[t]{2}{*}{$\mathrm{E}$} \\
\hline & & & Effluent wastewater ${ }^{\mathrm{AS}}$ & $0.042-0.098$ & $0.072-0.210$ & $0.63-0.68$ & \\
\hline & \multirow[t]{2}{*}{ UK } & \multirow[t]{2}{*}{ UPLC-MS/MS } & Effluent wastewater ${ }^{\mathrm{TF}}$ & 0.24 & 0.46 & 0.66 & \multirow[t]{2}{*}{$\mathrm{F}$} \\
\hline & & & River water & $<0.263$ & $<0.114$ & - & \\
\hline & \multirow[t]{6}{*}{ Australia } & \multirow[t]{6}{*}{ GC-MS/MS } & Influent wastewater ${ }^{\text {FASOS discharge }}$ & 0.065 & 0.179 & 0.73 & \multirow[t]{6}{*}{ G } \\
\hline & & & Effluent wastewater ${ }^{\mathrm{MBR}}$ & 0.007 & 0.007 & 0.50 & \\
\hline & & & Effluent wastewater ${ }^{\mathrm{AS}}$ & 0.005 & 0.005 & 0.50 & \\
\hline & & & Effluent wastewater ${ }^{\mathrm{AS}-\mathrm{BNR}}$ & $<0.001$ & $<0.001$ & - & \\
\hline & & & $\begin{array}{l}\text { Creek upstream of wastewater } \\
\text { discharge }\end{array}$ & 0.049 & 0.121 & 0.71 & \\
\hline & & & $\begin{array}{l}\text { Creek downstream of wastewa- } \\
\text { ter discharge }\end{array}$ & $0.015-0.026$ & $0.023-0.086$ & $0.60-0.77$ & \\
\hline & Australia & GC-EI-MS/MS & Influent wastewater ${ }^{\mathrm{MBR}}$ & $0.444-2.92$ & $5.27-39.5$ & $0.88-0.94$ & $\mathrm{H}$ \\
\hline & & & Effluent wastewater ${ }^{\mathrm{MBR}}$ & $0.006-0.017$ & $0.004-0.011$ & $0.38-0.40$ & \\
\hline & Spain & GC-EI-MS & 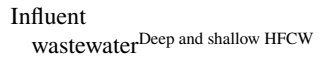 & n.a & n.a & 0.73 & I \\
\hline & & & Effluent wastewater ${ }^{\text {Deep HFCW }}$ & n.a & n.a & 0.76 & \\
\hline & & & Effluent wastewater ${ }^{\text {Shallow HFCW }}$ & n.a & n.a & 0.65 & \\
\hline & & & $\begin{array}{l}\text { Influent } \\
\text { wastewater }{ }^{\mathrm{VFCW} \text { (unsaturated) }}\end{array}$ & n.a & n.a & 0.90 & \\
\hline & & & 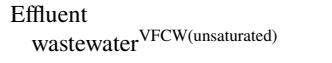 & n.a & n.a & 0.72 & \\
\hline & & & Effluent wastewater ${ }^{\mathrm{SF} \text { (unsaturated) }}$ & n.a & n.a & 0.71 & \\
\hline & & & Influent wastewater ${ }^{\mathrm{VFCW} \text { (saturated) }}$ & n.a & n.a & 0.90 & \\
\hline & & & Effluent wastewater ${ }^{\mathrm{VFCW} \text { (Saturated) }}$ & n.a & n.a & 0.60 & \\
\hline & & & Effluent wastewater ${ }^{\mathrm{SF}(\text { saturated) }}$ & n.a & n.a & 0.73 & \\
\hline & & & Influent wastewater ${ }^{\mathrm{AS}}$ & n.a & n.a & 0.88 & \\
\hline & & & Effluent wastewater ${ }^{\mathrm{AS}}$ & n.a & n.a & 0.64 & \\
\hline & Switzerland & GC-EI-MS/MS & Influent wastewater ${ }^{\text {biological }}$ & $0.990-3.300^{\mathrm{a}}$ & & $0.85-0.89$ & $\mathrm{~J}$ \\
\hline & & & Effluent wastewater ${ }^{\text {biological }}$ & $0.002-0.081^{\mathrm{a}}$ & & $0.47-0.67$ & \\
\hline & & & River/Lake water & $<0.0002-0.008^{\mathrm{a}}$ & & $0.41-0.81$ & \\
\hline Ketoprofen & UK & LC-MS/MS & Influent wastewater ${ }^{\mathrm{TF}}$ & 0.032 & 0.039 & 0.54 & A \\
\hline & & & Effluent wastewater ${ }^{\mathrm{TF}}$ & 0.003 & 0.006 & 0.65 & \\
\hline & & & Influent wastewater ${ }^{\mathrm{AS}}$ & 0.012 & 0.016 & 0.58 & \\
\hline & & & Effluent wastewater ${ }^{\mathrm{AS}}$ & $<0.0005$ & $<0.0005$ & - & \\
\hline & & & Influent wastewater ${ }^{\mathrm{SBR}}$ & $<0.013$ & $<0.013$ & - & \\
\hline & & & Effluent wastewater ${ }^{\mathrm{SBR}}$ & 0.114 & 0.200 & 0.64 & \\
\hline & Spain & LC-MS/MS & Influent wastewater ${ }^{\mathrm{AS}}$ & $0.088-0.240$ & $0.121-0.510$ & $0.54-0.68$ & $\mathrm{E}$ \\
\hline & & & Effluent wastewater ${ }^{\mathrm{AS}}$ & $0.024-0.086$ & $0.038-0.177$ & $0.61-0.68$ & \\
\hline & Australia & GC-EI-MS/MS & Influent wastewater ${ }^{\mathrm{MBR}}$ & $0.065-12.0$ & $0.084-15.3$ & $0.56-0.60$ & $\mathrm{H}$ \\
\hline & & & Effluent wastewater ${ }^{\mathrm{MBR}}$ & $0.001-0.005$ & $0.002-0.006$ & $0.54-0.68$ & \\
\hline Naproxen & UK & LC-MS/MS & Influent wastewater ${ }^{\mathrm{TF}}$ & 0.311 & 24.3 & 0.99 & A \\
\hline & & & Effluent wastewater ${ }^{\mathrm{TF}}$ & 0.391 & 4.12 & 0.92 & \\
\hline & & & Influent wastewater ${ }^{\mathrm{AS}}$ & $<0.010$ & 32.9 & $>0.99$ & \\
\hline & & & Effluent wastewater ${ }^{\mathrm{AS}}$ & $<0.004$ & 0.116 & $>0.97$ & \\
\hline & & & Influent wastewater ${ }^{\mathrm{SBR}}$ & $<0.010$ & 17.0 & $>0.99$ & \\
\hline & & & Effluent wastewater ${ }^{\mathrm{SBR}}$ & $<0.004$ & 0.579 & $>0.99$ & \\
\hline
\end{tabular}


Table 3 (continued)

\begin{tabular}{|c|c|c|c|c|c|c|c|}
\hline Drug & Country & Analysis method & Sampled matrix & $R(-)$-enantiomer $\left(\mu \mathrm{g} \mathrm{L}^{-1}\right)$ & $S(+)$-enantiomer $\left(\mu \mathrm{g} \mathrm{L}^{-1}\right)$ & $\mathrm{EF}$ & References \\
\hline & & & $\begin{array}{l}\text { River upstream of wastewater } \\
\text { discharge }\end{array}$ & 0.09 & 0.35 & 0.92 & \\
\hline & & & $\begin{array}{l}\text { River downstream of wastewa- } \\
\text { ter discharge }\end{array}$ & 0.01 & 0.35 & 0.97 & \\
\hline & China & LC-MS/MS & River water & $<0.0004-0.002$ & $<0.0004-0.043$ & 0.93 & B \\
\hline & UK & LC-MS/MS & Influent wastewater ${ }^{\mathrm{AS}}$ & $<0.011$ & 0.37 & $>0.99$ & K \\
\hline & & & Effluent wastewater ${ }^{\mathrm{AS}}$ & $<0.014$ & $<0.013$ & - & \\
\hline & & & River water & $<0.008$ & $<0.007$ & - & \\
\hline & Europe & UHSFC-MS/MS & Influent wastewater & $<0.233$ & 4.75 & $>0.95$ & $\mathrm{D}$ \\
\hline & & & Effluent wastewater & $<0.539$ & 0.95 & $>0.64$ & \\
\hline & UK & UPLC-MS/MS & Effluent wastewater ${ }^{\mathrm{TF}}$ & 0.09 & 1.33 & 0.94 & $\mathrm{~F}$ \\
\hline & & & River water & $<0.008$ & 0.136 & $>0.98$ & \\
\hline & Spain & LC-MS/MS & Influent wastewater ${ }^{\mathrm{AS}}$ & $0.018-0.030$ & $1.049-3.172$ & $0.98-0.99$ & E \\
\hline & & & Effluent wastewater ${ }^{\mathrm{AS}}$ & $0.009-0.022$ & $0.175-0.481$ & $0.93-0.96$ & \\
\hline & Japan & LC-MS/MS & Influent wastewater ${ }^{\mathrm{AS}}$ & $0.03-0.43^{\mathrm{a}}$ & & $>0.99$ & $\mathrm{~L}$ \\
\hline & & & Effluent wastewater ${ }^{\mathrm{AS}}$ & $0.01-0.11^{\mathrm{a}}$ & & $0.88-0.91$ & \\
\hline & & & River water & $0.08^{\mathrm{a}}$ & & $0.84-0.98$ & \\
\hline & Australia & GC-MS/MS & Influent wastewater ${ }^{\text {FASOS discharge }}$ & $<0.001$ & 0.024 & $>0.96$ & G \\
\hline & & & Effluent wastewater ${ }^{\mathrm{MBR}}$ & 0.001 & 0.008 & 0.90 & \\
\hline & & & Effluent wastewater ${ }^{\mathrm{AS}}$ & 0.003 & 0.033 & 0.92 & \\
\hline & & & Effluent wastewater ${ }^{\mathrm{AS}-\mathrm{BNR}}$ & 0.007 & 0.013 & 0.65 & \\
\hline & & & $\begin{array}{l}\text { Creek upstream of wastewater } \\
\text { discharge }\end{array}$ & $<0.001$ & 0.025 & $>0.96$ & \\
\hline & & & $\begin{array}{l}\text { Creek downstream of wastewa- } \\
\text { ter discharge }\end{array}$ & $<0.001$ & $0.055-0.604$ & $>0.98$ & \\
\hline & Australia & GC-EI-MS/MS & Influent wastewater ${ }^{\mathrm{MBR}}$ & $0.010-0.032$ & $0.827-67.6$ & 0.99 & $\mathrm{H}$ \\
\hline & & & Effluent wastewater ${ }^{\mathrm{MBR}}$ & $0.002-0.014$ & $0.025-0.161$ & $0.86-0.94$ & \\
\hline & Spain & GC-EI-MS & $\begin{array}{l}\text { Influent } \\
\text { wastewater }{ }^{\text {Deep and shallow HFCW }}\end{array}$ & n.a & n.a & 0.89 & I \\
\hline & & & Effluent wastewater ${ }^{\text {Deep HFCW }}$ & n.a & n.a & 0.82 & \\
\hline & & & Effluent wastewater ${ }^{\text {Shallow } \mathrm{HFCW}}$ & n.a & n.a & 0.72 & \\
\hline & & & $\begin{array}{l}\text { Influent } \\
\text { wastewater }^{\mathrm{VFCW} \text { (unsaturated) }}\end{array}$ & n.a & n.a & 0.90 & \\
\hline & & & $\begin{array}{l}\text { Effluent } \\
\text { wastewater } \\
\mathrm{VFCW} \text { (unsaturated) }\end{array}$ & n.a & n.a & 0.71 & \\
\hline & & & Effluent wastewater ${ }^{\mathrm{SF}(\text { unsaturated) }}$ & n.a & n.a & 0.76 & \\
\hline & & & Influent wastewater ${ }^{\mathrm{VFCW} \text { (saturated) }}$ & n.a & n.a & 0.90 & \\
\hline & & & Effluent wastewater ${ }^{\mathrm{VFCW}(\text { saturated) }}$ & n.a & n.a & 0.78 & \\
\hline & & & Effluent wastewater ${ }^{\mathrm{SF}(\text { saturated) }}$ & n.a & n.a & 0.79 & \\
\hline & & & Influent wastewater ${ }^{\mathrm{AS}}$ & n.a & n.a & 0.88 & \\
\hline & & & Effluent wastewater ${ }^{\mathrm{AS}}$ & n.a & n.a & 0.86 & \\
\hline \multirow[t]{3}{*}{ Flurbiprofen } & China & LC-MS/MS & Influent wastewater & 0.079 & 0.088 & 0.54 & $\mathrm{C}$ \\
\hline & & & Effluent wastewater & $<0.013$ & $<0.014$ & - & \\
\hline & & & River water & 0.032 & 0.04 & 0.55 & \\
\hline
\end{tabular}

Key: AS, activated sludge; AS-BNR, activated sludge-biological nitrogen removal; EF, enantiomeric fraction; FASOS, fuller avenue sewage overflow structure; GC-EI-MS, gas chromatography-electron impact-mass spectrometry; GC-EI-MS/MS, gas chromatography-electron impact-tandem mass spectrometry; GC-MS/MS, gas chromatography-tandem mass spectrometry; HFCW, horizontal subsurface-flow constructed wetlands; LC-MS/MS, liquid chromatography-tandem mass spectrometry; MBR, membrane bioreactor; n.a., not available; SF, sand filter; TF, trickling filter beds; UHSFC-MS/MS, ultra-high supercritical fluid chromatography-tandem mass spectrometry; UPLC-MS/MS, ultraperformance liquid chromatography-tandem mass spectrometry; VFCW, vertical-flow constructed wetlands

${ }^{\text {a }}$ Total enantiomeric concentration; ${ }^{\mathrm{A}}$ Camacho-Muñoz et al. (2019); ${ }^{\mathrm{B}} \mathrm{Ma}$ et al. (2019); ${ }^{\mathrm{C}}$ Wang et al. (2018); ${ }^{\mathrm{D}} \mathrm{Camacho-Muñoz} \mathrm{et} \mathrm{al.} \mathrm{(2016);}$ ${ }^{\mathrm{E}}$ Caballo et al. (2015a); ${ }^{\mathrm{F}}$ Camacho-Muñoz and Kasprzyk-Hordern (2015); ${ }^{\mathrm{G}}$ Khan et al. (2014); ${ }^{\mathrm{H}}$ Hashim et al. (2013); ${ }^{\mathrm{I}}$ Matamoros et al. (2009); ${ }^{\mathrm{J}}$ Buser et al. (1999); ${ }^{\mathrm{K}}$ Camacho-Muñoz and Kasprzyk-Hordern (2017); ${ }^{\mathrm{L}}$ Suzuki et al. (2014) 
observed including chiral NSAIDs (Camacho-Muñoz et al. 2012; Kasprzyk-Hordern et al. 2009; Petrie et al. 2015; Roberts and Thomas 2006; Verlicchi et al. 2012). Removal of NSAIDs can be variable between wastewater treatment plants and individual drugs. For example, higher removal of naproxen has been reported by activated sludge over trickling filters (Kasprzyk-Hordern et al. 2009). Furthermore, five full-scale activated sludge wastewater treatment plants in Japan showed ibuprofen removals were greater than $90 \%$, whereas average removals of both naproxen and ketoprofen were $45 \%$ (Nakada et al. 2006). The removal of NSAIDs is mainly attributed to biodegradation (Matamoros et al. 2009; Nakada et al. 2006; Onesios et al. 2009; Patel et al. 2019; Petrie et al. 2014; Verlicchi et al. 2012), whereby the parent drugs are transformed into a metabolite or degradation product, or is mineralized (Patel et al. 2019). Importantly, microbial processes during wastewater treatment can result in enantioselectivity.

During activated sludge, trickling filter and membrane bioreactor treatment, removal favours $S(+)$-ibuprofen causing a reduction of enantiomeric fraction (Buser et al. 1999; Caballo et al. 2015a; Hashim et al. 2013; Khan et al. 2014; Matamoros et al. 2009) (Table 3). Ibuprofen enantiomeric fractions in effluent wastewater are in the range 0.50-0.71 with concentrations less than $1 \mu \mathrm{g} \mathrm{L}{ }^{-1}$ (Table 3). Matamoros et al. (2009) also investigated the enantiomeric behaviour of ibuprofen during treatment by horizontal sub-surface and vertical flow constructed wetlands and sand filters. Under aerobic conditions, preferential removal of $S(+)$-ibuprofen was also observed. However, such systems operated under prevailing anaerobic conditions resulted in ibuprofen removal not being enantioselective (Matamoros et al. 2009). Therefore, redox conditions are important as different bacterial consortia are active under aerobic and anaerobic conditions.

Only limited data exist on the enantioselective behaviour of ketoprofen during wastewater treatment (Table 3). No significant changes to ketoprofen enantiomeric fractions were reported by activated sludge or membrane bioreactor treatment despite considerable removal (Caballo et al. 2015a; Hashim et al. 2013). However, Camacho-Muñoz et al. (2019) reported an enrichment of $S(+)$-ketoprofen during trickling filter treatment. Influent and effluent enantiomeric fractions were 0.54 and 0.65 , respectively (Table 3 ). Differences in enantioselectivity during activated sludge and trickling filter treatment has been observed for other drugs such as 3,4-methylenedioxymethamphetamine due to different consortia of microorganisms between process types (Kasprzyk-Hordern and Baker 2012).

Enantioselectivity has been reported for naproxen during wastewater treatment by trickling filters, activated sludge, membrane bioreactors, constructed wetlands and sand filters (Caballo et al. 2015a; Camacho-Muñoz et al. 2019; Khan et al. 2014; Matamoros et al. 2009; Suzuki et al. 2014). An increase in the relative concentration of $R(-)$-naproxen resulted in enantiomeric fractions within wastewater effluents being in the range 0.65-0.98 (Table 3). Khan et al. (2014) reported $R(-)$-naproxen concentrations in wastewater effluents up to $0.007 \mu \mathrm{g} \mathrm{L}^{-1}$, whereas no measurable concentration was present in influent wastewater. Due to this increased concentration, it was postulated that chiral inversion of $S(+)$ naproxen took place (Khan et al. 2014). The enantioselectivity of naproxen is reported to be similar under both prevailing aerobic and anaerobic conditions (Matamoros et al. 2009). Therefore, it was also suggested that the change in enantiomeric fraction observed for naproxen during wastewater treatment could be used to distinguish between treated and untreated sources of wastewater in the environment (Khan et al. 2014). Similar observations have been made for the beta-blocker propranolol (Fono and Sedlak 2005) and the stimulant amphetamine (Ramage et al. 2019).

\section{Spiked wastewater microcosms for fate evaluation}

Understanding the fate processes responsible for enantioselectivity, i.e. degradation or inversion, during full-scale wastewater treatment is challenging. Batch and flow through microcosm studies using spiked enantiomer concentrations enable a greater understanding of these processes whereby operational variables can be closely controlled. Several studies have reported limited or no enantioselectivity of ibuprofen in batch studies of diluted activated sludge (Escuder-Gilabert et al. 2018) or laccase enzyme obtained from a culture of the white rot fungus Pleurotus ostreatus (Nguyen et al. 2017). Furthermore, no enantioselectivity was observed in constructed wetlands treating synthetic or real wastewater under predominantly anaerobic conditions at dissolved oxygen concentrations less than $0.5 \mathrm{mg} \mathrm{L}^{-1}$ (Matamoros et al. 2009).

In a continuous flow membrane bioreactor system treating synthetic wastewater containing $R / S( \pm)$-ibuprofen, an enrichment of $R(-)$-ibuprofen was observed (Hashim et al. 2011). In this study, the enantiomeric fraction reduced from 0.50-0.54 to 0.31-0.44 (Table 4). However, as ibuprofen was used as the racemate, it was not possible to establish the process(es) responsible for enantioselectivity, i.e. degradation or inversion. Nguyen et al. (2017) investigated the behaviour of individual ibuprofen enantiomers separately in a continuous flow enzymatic membrane bioreactor dosed with laccase. The enantiomeric fraction increased from 0.01 to 0.18 in studies of $R(-)$-ibuprofen only and reduced from 0.99 to 0.75 in studies of $S(+)$-ibuprofen only (Table 4 ). These observations confirmed that ibuprofen enantiomers can undergo bidirectional inversion during wastewater treatment (Nguyen et al. 2017).

The mechanism(s) of inversion or microbes responsible for inversion during wastewater treatment remain poorly understood. Fungi including Verticillium lecanii are 


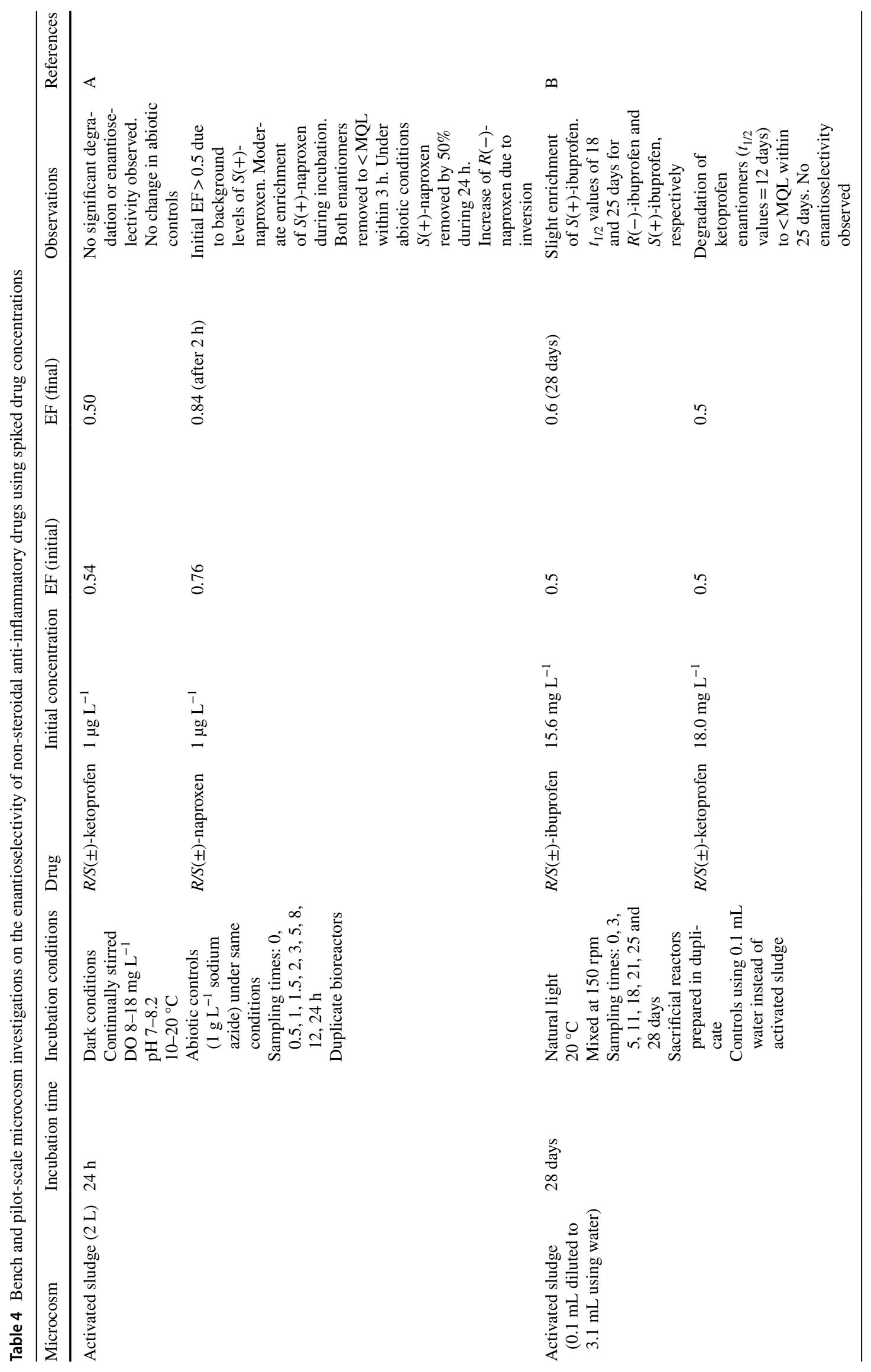




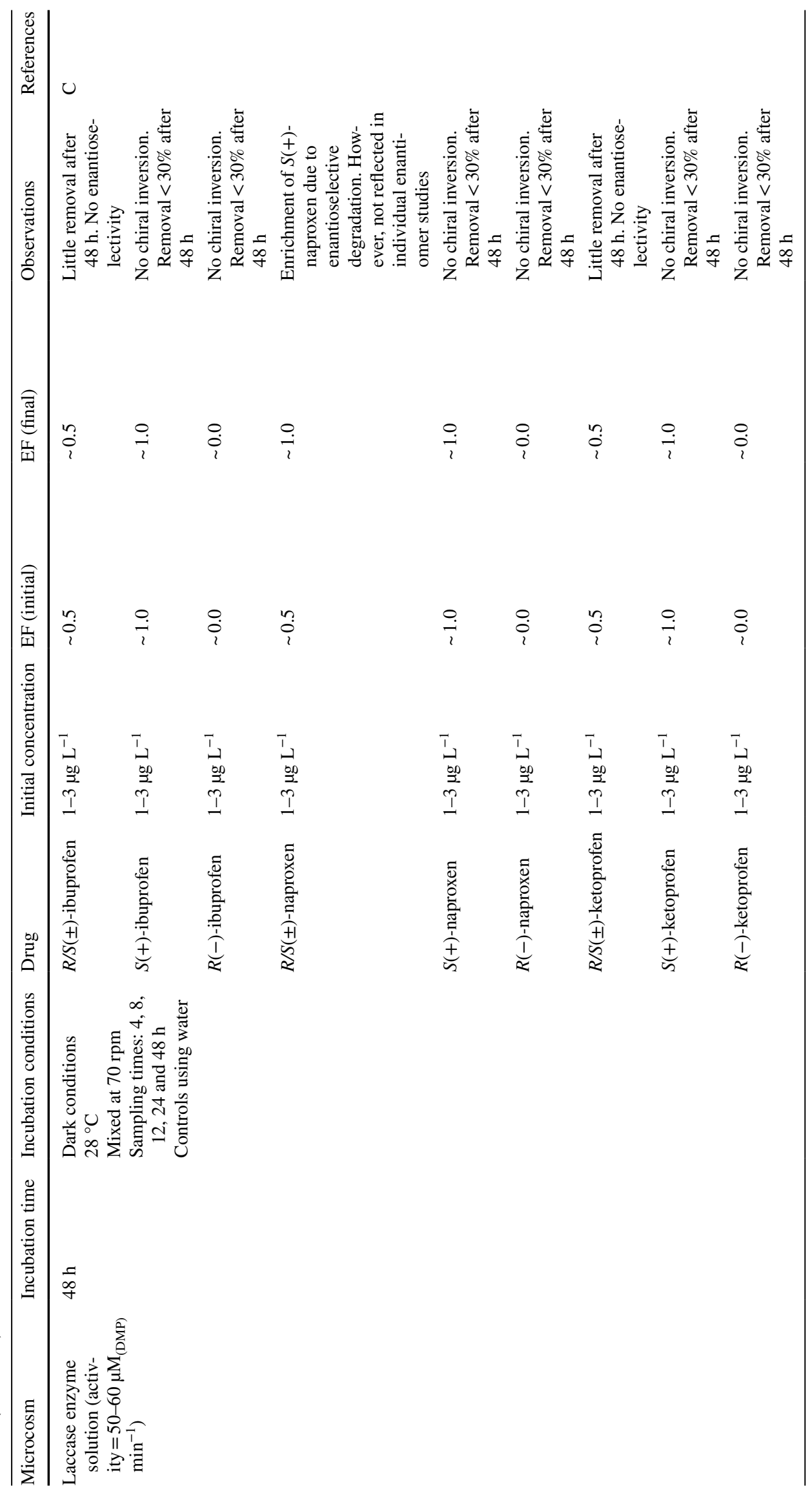




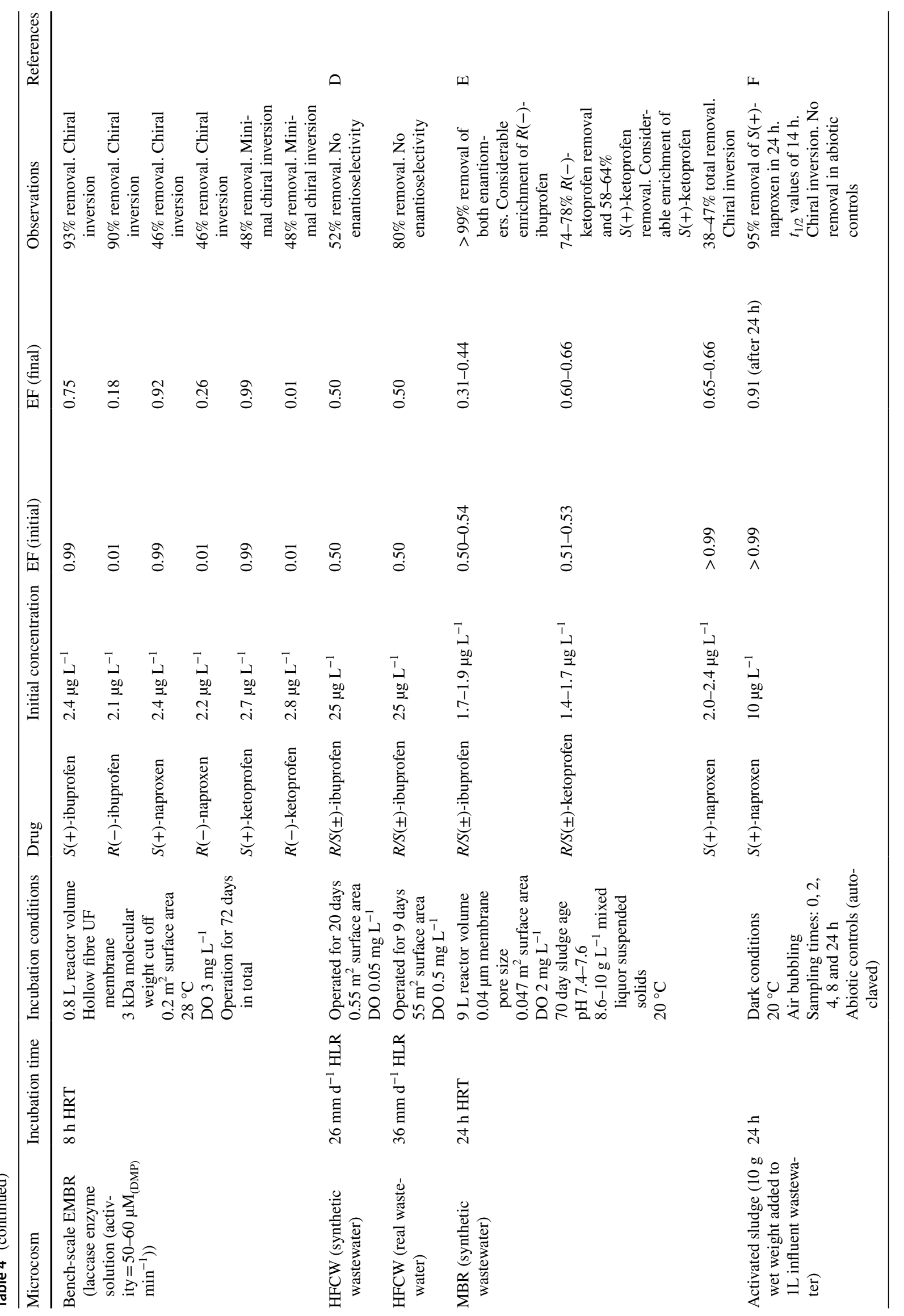




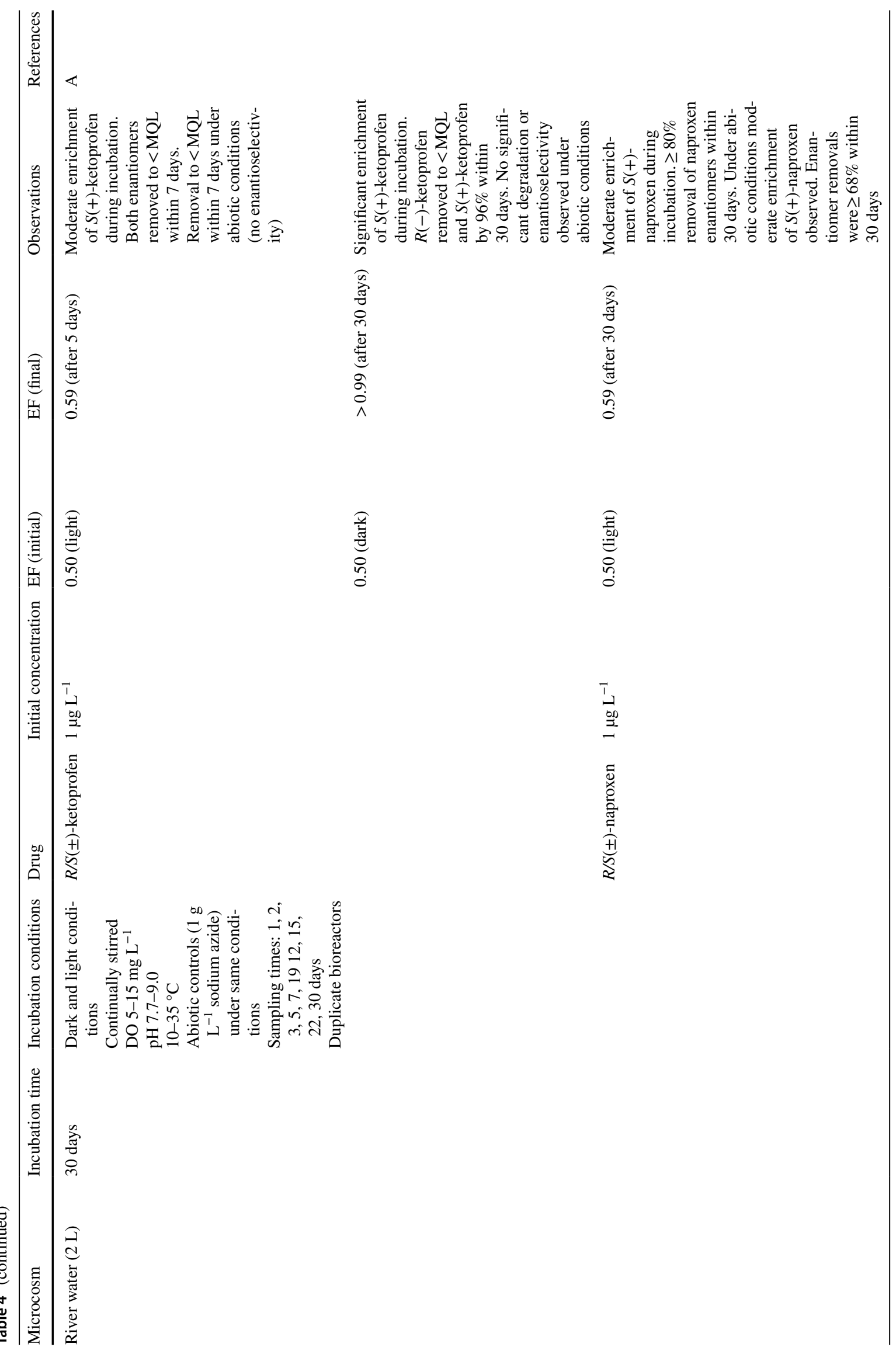




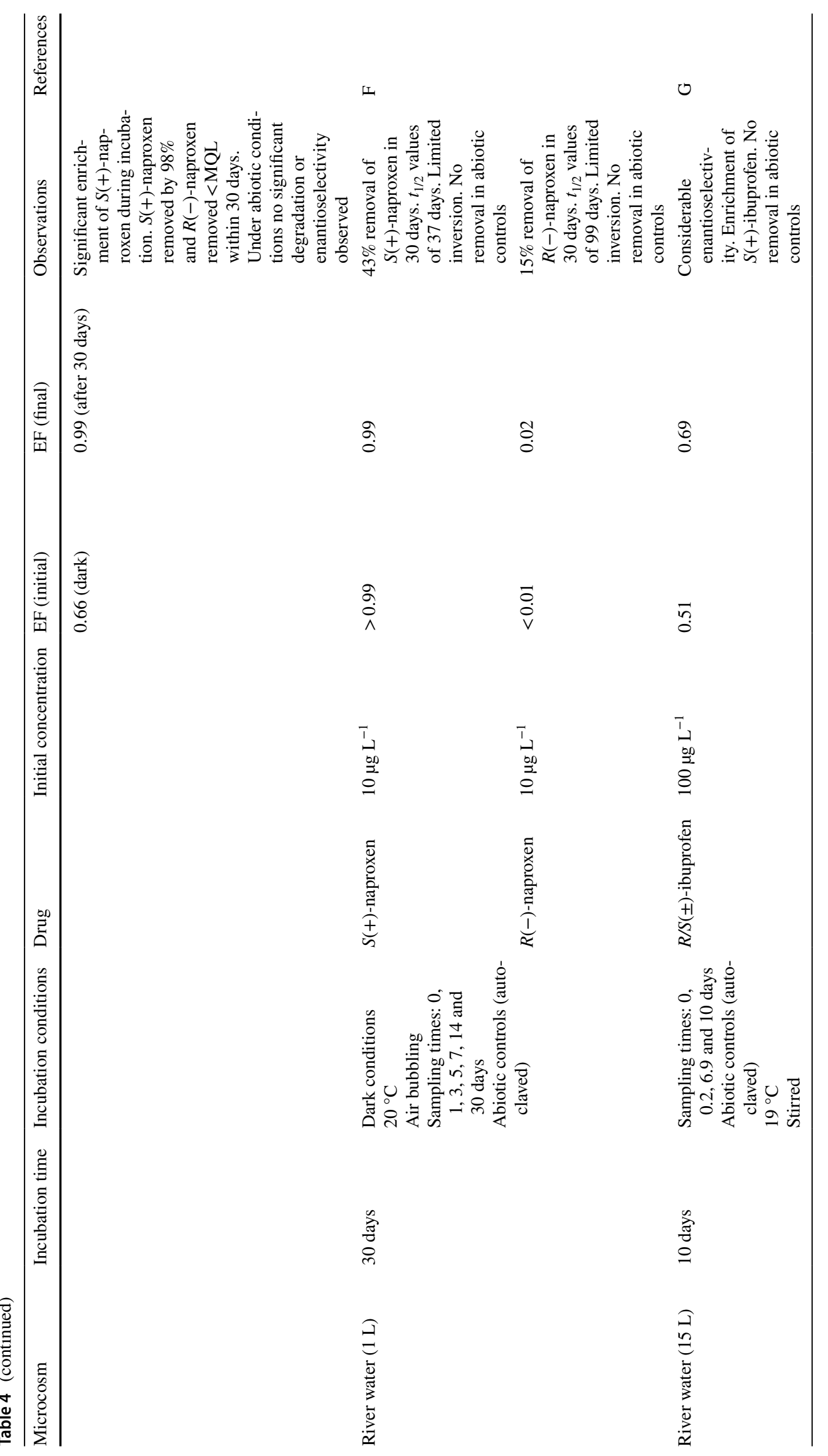




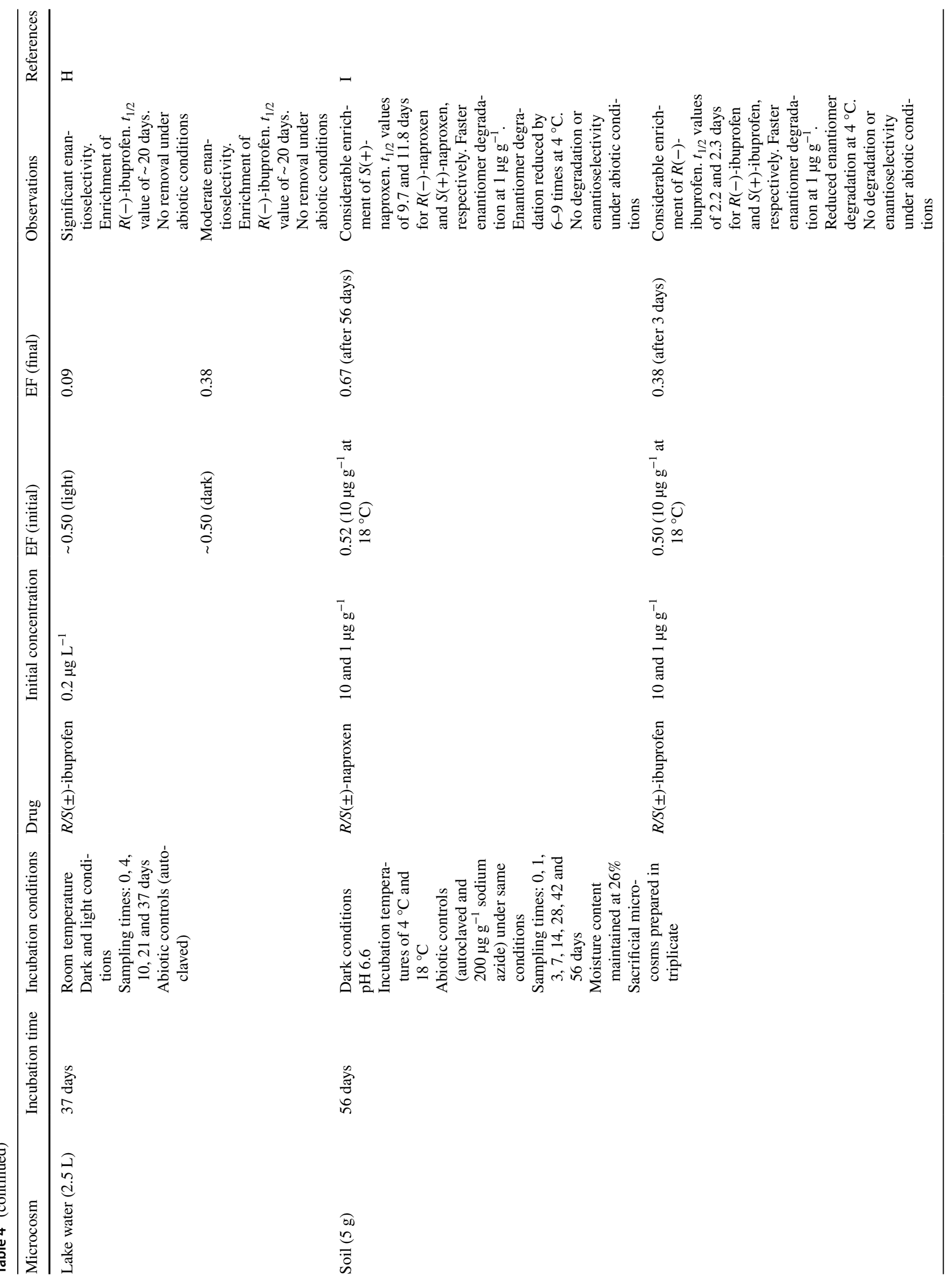




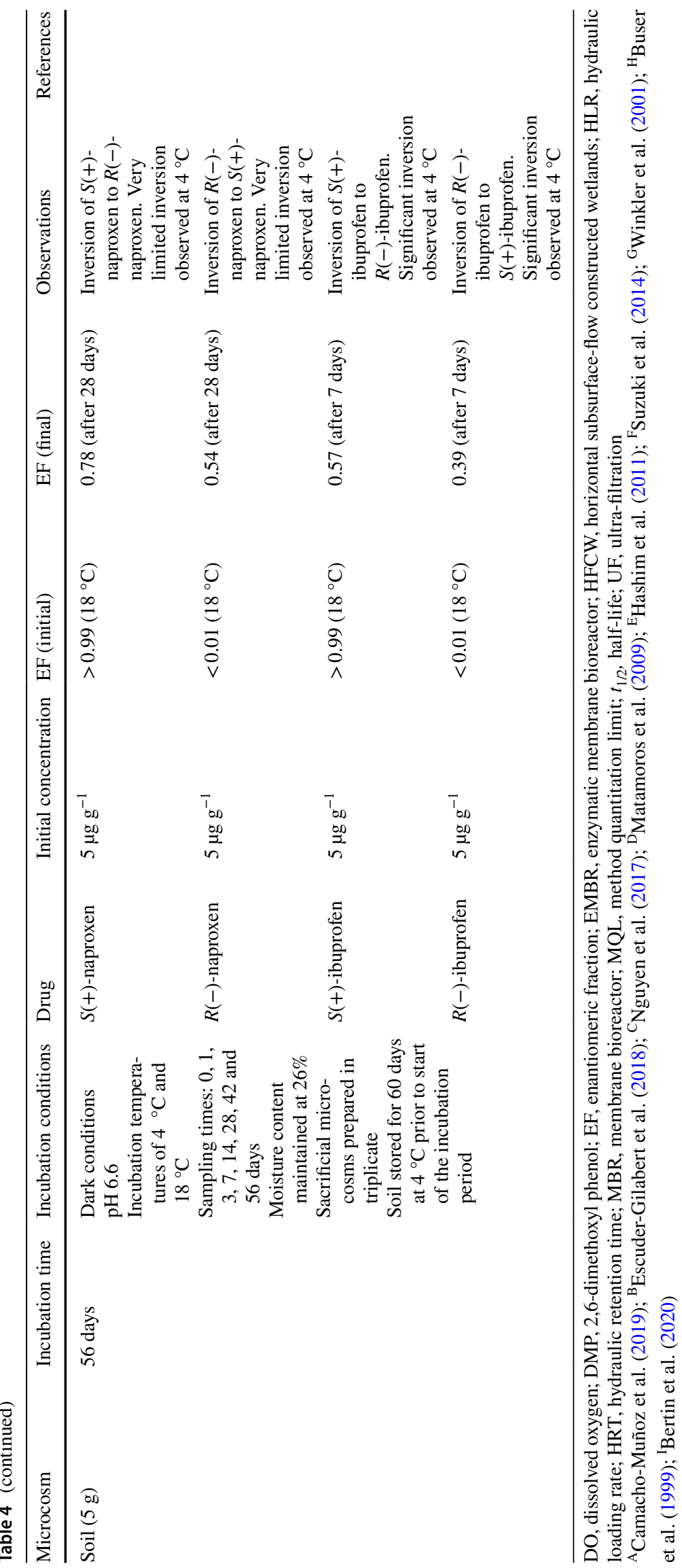


suggested to invert ibuprofen enantiomers in both directions, favouring the formation of $S(+)$-ibuprofen (Thomason et al. 1997). Nocardia bacteria can invert $S(+)$-ibuprofen to $R(-)$ ibuprofen (Mitsukura et al. 2002). Inversion in this instance is thought to involve several enzymes via a similar mechanism observed in rat liver (Kato et al. 2004, 2003) (Fig. 2). However, considering the diversity in the microbial community present in wastewater, several inversion pathways are possible.

No enantioselectivity in the degradation of $R / S( \pm)$ ketoprofen has been reported in activated sludge or laccase enzyme batch studies (Camacho-Muñoz et al. 2019; Escuder-Gilabert et al. 2018; Nguyen et al. 2017). However, minimal bidirectional chiral inversion was observed in a continuous flow enzymatic membrane bioreactor treating synthetic wastewater containing individual ketoprofen enantiomers (Nguyen et al. 2017). In a further membrane bioreactor study, $R / S( \pm)$-ketoprofen was enriched with $S(+)$ ketoprofen whereby a racemic enantiomeric fraction was increased to 0.60-0.66 (Hashim and Khan 2011) (Table 4).

Studies using enantiomerically pure $S(+)$-naproxen have reported inversion to $R(-)$-naproxen in activated sludge and membrane bioreactor microcosms (Hashim et al. 2011; Suzuki et al. 2014). Suzuki et al. (2014) found no inversion in autoclaved abiotic controls confirming the inversion process is driven biologically. Activated sludge microcosms spiked with $R / S( \pm)$-naproxen provided evidence of $S(+)$ naproxen inversion (Camacho-Muñoz et al. 2019). Nguyen et al. (2017) postulated enantioselective degradation of $R / S( \pm)$-naproxen occurred in laccase enzyme batch studies. Within $4 \mathrm{~h}, R(-)$-naproxen was removed to below the MDL, whereas $S(+)$-naproxen remained largely unchanged for $24 \mathrm{~h}$ (Nguyen et al. 2017). Enantioselective degradation was proposed to be responsible because no inversion was observed in single enantiomer microcosms incubated in the same way. However, in these single enantiomer studies limited degradation was observed (Table 4). Therefore, further studies are needed to confirm enantioselective degradation as the main driver to the changes in enantiomeric fraction observed in the racemic microcosm. Nguyen et al. (2017) also demonstrated that naproxen underwent bidirectional inversion in a continuous flow enzymatic membrane bioreactor dosed with laccase. In single enantiomer studies, the formation of $S(+)$-naproxen was favoured over $R(-)$-naproxen (Table 4). However, it is challenging to identify whether this is a result of the inversion process(es) being enantioselective, i.e. inversion rates of enantiomers are different, or it is a result of enantioselective degradation, i.e. degradation rates of enantiomers are different, or it is a combination of both processes being enantioselective.

\section{Occurrence and behaviour in receiving environments}

\section{Aquatic environment}

The $R / S( \pm$ )-ibuprofen concentration in surface waters is less than $1 \mu \mathrm{g} \mathrm{L}^{-1}$ (Table 3). The enantiomeric fraction is typically in the range 0.60-0.81 (Buser et al. 1999; Khan et al. 2014; Ma et al. 2019; Wang et al. 2018), similar to those enantiomeric fractions observed in effluent wastewater (Table 3 ). However, an enrichment of $R(-)$-ibuprofen with a corresponding enantiomeric fraction of 0.41 has been reported (Buser et al. 1999). In river water microcosms, considerable enantioselectivity was observed for $R / S( \pm)$-ibuprofen over 10 days (Winkler et al. 2001). Enrichment of $S(+)$-ibuprofen was observed with a change in enantiomeric fraction from 0.51 to 0.69 (Table 4). No degradation or enantioselectivity was observed in abiotic controls. On the other hand, Buser et al. (1999) noted an enrichment of $R(-)$-ibuprofen in lake water microcosms incubated for 37 days (Table 4). These studies demonstrate the diverse enantiospecific behaviour of ibuprofen in the environment. In both studies, ibuprofen was spiked as the racemate and it was not possible to establish whether or not chiral inversion took place.

Currently, no data exist on the enantiomeric composition of ketoprofen in surface waters. $R / S( \pm)$-ketoprofen has been found to degrade rapidly in river water microcosms (Camacho-Muñoz et al. 2019). Both biodegradation and photolysis contributed to ketoprofen removal from river water microcosms. In biotic and abiotic microcosms under light exposure, the total ketoprofen removal after 5 days was 98\% (Camacho-Muñoz et al. 2019). Under biotic conditions (both light and dark exposure), an enrichment of the pharmacologically active enantiomer $S(+)$-ketoprofen was observed (Table 4).

Naproxen has been reported in surface waters up to $0.36 \mu \mathrm{g} \mathrm{L}^{-1}$ with enantiomeric fractions in the range 0.84-0.98 (Table 3). Most studies report the presence of $R(-)$-naproxen due to the inversion observed during wastewater treatment. River water microcosm studies spiked with $R / S( \pm)$-naproxen revealed an enrichment of $S(+)$-naproxen during incubation (Camacho-Muñoz et al. 2019). Suzuki et al. (2014) studied the enantiospecific behaviour of naproxen in river water microcosms spiked with individual enantiomers. Very little inversion was observed for both enantiomers during 30-day incubation. However, removal was considerably different between $S(+)$-naproxen and $R(-)$ naproxen microcosms (Table 4). The half-lives of $S(+)$-naproxen and $R(-)$-naproxen were 37 and 99 days, respectively (Suzuki et al. 2014). The lack of inversion and considerably different removal rates between enantiomers demonstrates enantioselective degradation of naproxen in river water. 


\section{Terrestrial environment}

The application of biosolids as fertilizer or irrigation with reclaimed wastewater can result in the introduction of chiral NSAIDs to agricultural soils (Albero et al. 2014; Radjenović et al. 2009a). Both naproxen and ibuprofen have been reported in amended soils (Biel-Maeso et al. 2018; Gibson et al. 2010). However, no data exist on the enantiomeric composition of these drugs in soil. Nevertheless, spiked microcosm studies have revealed important information on the behaviour of ibuprofen and naproxen here. Individual enantiomer microcosms revealed both naproxen and ibuprofen are subject to bidirectional chiral inversion in soil (Bertin et al. 2020). $S(+)$-naproxen and $R(-)$-ibuprofen were found to be the more persistent enantiomers. For example, soil microcosms maintained at $18{ }^{\circ} \mathrm{C}$ and spiked with $R(-)$ naproxen with an initial enantiomeric fraction less than 0.01 increased to 0.54 after 28 days. Microcosms spiked with $S(+)$-naproxen, and an initial enantiomeric fraction greater than 0.99 reduced to 0.78 after 28 days (Fig. 3). However, it was not possible to conclude that this was due to different rates of inversion or degradation between enantiomers as these processes can take place simultaneously. Naproxen enantiomers were found to persist in soil for at least 56 days, whereas ibuprofen degraded completely within 28 days (Bertin et al. 2020). Enantioselectivity and enantiomer removal were significantly reduced in soils incubated at $4{ }^{\circ} \mathrm{C}$ (Fig. 3), which is an important consideration for cooler and temperate climates. No enantiomer removal or enantioselectivity was observed in abiotic controls.

\section{Enantiospecific toxicity}

The effects of drug enantiomers on target organisms such as humans are well understood. Yet, studies on the enantiospecific effects of drugs to non-target organisms are limited, particularly for the NSAIDs. Mennillo et al. (2018) reported the toxicity of $R / S( \pm)$-ketoprofen and the pharmacologically active $S(+)$-ketoprofen to bacteria, algae and zooplankton. $S(+)$-ketoprofen was more potent to algae than the racemate at the same concentration. To demonstrate, median effect concentrations $\left(\mathrm{EC}_{50}\right.$-concentration that the toxicological response is halfway between a normal and maximum response) towards the microalgae Pseudokirchneriella subcapitata (growth inhibition after $96 \mathrm{~h}$ ) were 240 and

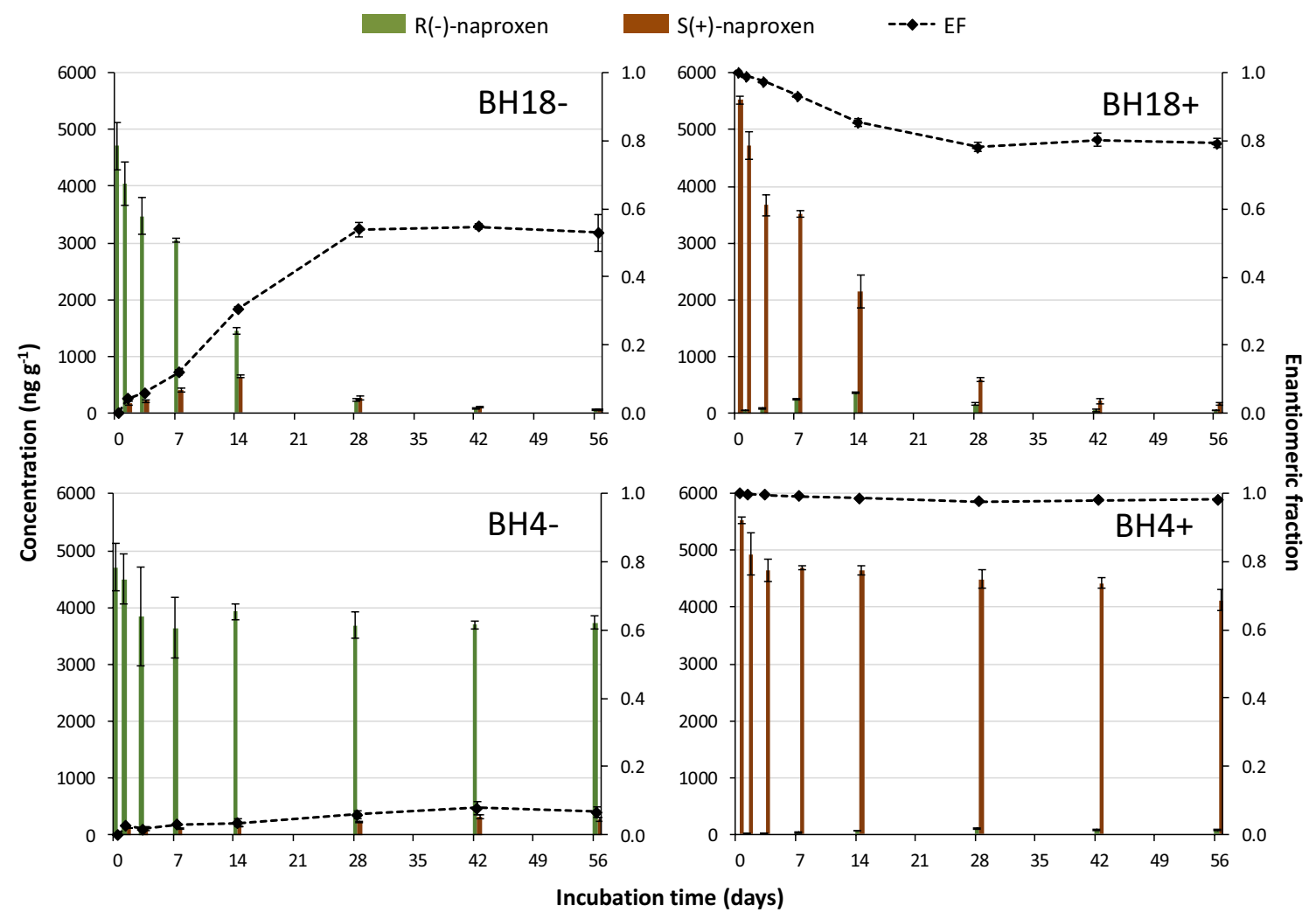

Fig. 3 Concentration of $R(-)$-naproxen and $S(+)$-naproxen and the corresponding enantiomeric fractions in soil microcosms spiked with individual naproxen enantiomers. Reproduced with permission (Bertin et al. 2020). Key: BH18-, biotic high spike level of $R(-)$-naproxen

$18{ }^{\circ} \mathrm{C}$ microcosm; BH18+, biotic high spike level of $S(+)$-naproxen $18{ }^{\circ} \mathrm{C}$ microcosm; BH4-, biotic high spike level of $R(-)$-naproxen 4 ${ }^{\circ} \mathrm{C}$ microcosm; BH4+, biotic high spike level of $S(+)$-naproxen $4{ }^{\circ} \mathrm{C}$ microcosm 
$66 \mu \mathrm{g} \mathrm{L}^{-1}$ for $R / S( \pm)$-ketoprofen and $S(+)$-ketoprofen, respectively (Mennillo et al. 2018) (Table 5). Furthermore, a no observed effect concentration (NOEC) of $4 \mu \mathrm{g} \mathrm{L}^{-1}$ has been reported for $S(+)$-ketoprofen towards $P$. subcapitata (7 days, growth) (Mennillo et al. 2018). This could be significant considering ketoprofen in wastewater effluents is enriched with $S(+)$-ketoprofen with enantiomeric fractions in the range $0.54-0.68$ (Table 4). However, no toxicity testing was undertaken on the pharmacologically less active $R(-)$-ketoprofen.

Neale et al. (2019) investigated the toxicity of $S(+)$ ketoprofen and $R(-)$-ketoprofen using a battery of ecotoxicity bioassays including bacteria, algae and fish cells. The photosystem inhibition of P. subcapitata $\left(\mathrm{EC}_{10}, 24 \mathrm{~h}\right)$ was greater than $16.7 \mathrm{mg} \mathrm{L}^{-1}$ for both enantiomers. Greatest enantiospecific differences were observed towards ethoxyresorufin-O-deethylase activity in fish cells (induction of cytochrome P450 1A enzymes which are important for xenobiotic metabolism (Fent 2001). The effect concentration causing an induction ratio of $1.5\left(\mathrm{EC}_{\mathrm{IR} 1.5}, 6 \mathrm{~h}\right)$ was $4.3 \mathrm{mg}$ $\mathrm{L}^{-1}$ for $R(-)$-ketoprofen and greater than $27.2 \mathrm{mg} \mathrm{L}^{-1}$ for $S(+)$-ketoprofen demonstrating at least 6.3 times potency difference between enantiomers (Neale et al. 2019). Here, the more potent enantiomer $R(-)$-ketoprofen was the less prevalent reported in the environment (Table 5).

Neale et al. (2019) also investigated the toxicity of individual flurbiprofen, ibuprofen and naproxen enantiomers in their study. Of the studied organisms, ibuprofen enantiomers exhibited toxicity towards the bacteria Photobacterium leiognathi. However, toxicity was not enantiospecific with $\mathrm{EC}_{50}$ values (\% inhibition, $30 \mathrm{~min}$ ) of 3.1 and $2.8 \mathrm{mg} \mathrm{L}^{-1}$ for $S(-)$-ibuprofen and $R(+)$-ibuprofen, respectively (Neale et al. 2019). However, studies on zebra fish (Danio rerio) have reported significant differences on lipid metabolites in brain tissue induced by $S(-)$-ibuprofen and $R(+)$-ibuprofen (Zhang et al. 2020). In this study, the test fish were exposed to environmentally relevant enantiomer concentrations $(5 \mu \mathrm{g}$ $\mathrm{L}^{-1}$ ) for 28 days. The authors concluded that ibuprofen can induce enantiospecific toxicity to aquatic organisms (Zhang et al. 2020).

Flurbiprofen showed greatest enantiospecific toxicity towards $P$. leiognathi albeit only 1.7 times difference between $S(+)$-flurbiprofen and $R(-)$-flurbiprofen $\left(\mathrm{EC}_{50}\right.$ 1.2 vs $2.1 \mathrm{mg} \mathrm{L}^{-1}$ ) (Neale et al. 2019). $S(+)$-naproxen was 2.5 times more active than $R(-)$-naproxen in the ethoxyresorufin-O-deethylase assay. However, the lowest $\mathrm{EC}_{50}$ values were reported for $P$. leiognath albeit with only slight enantiospecificity $\left(\mathrm{EC}_{50} 0.93\right.$ vs $0.75 \mathrm{mg} \mathrm{L}^{-1}$ ) (Table 5) (Neale et al. 2019). Considerably more toxicity data are available on $S(+)$-naproxen (without comparative data for $R(-)$-naproxen) as it the 'off-the-shelf' standard available (Table 5). This includes chronic toxicity data whereby the lowest NOEC to the crustacean Moina macrocopa (7 days, reproduction) was $0.3 \mathrm{mg} \mathrm{L}^{-1}$ (Kwak et al. 2018) (Table 5). Although $S(+)$-naproxen is the dominant enantiomer in the environment with enantiomeric fractions $0.84-0.98$, notable $R(-)$-naproxen concentrations are also present (Table 4). Therefore, toxicity assays also need undertaken on $R(-)$-naproxen.

\section{Recommendations for enantioselective studies and future perspectives}

In recent years, considerable improvements have been made on understanding the enantiospecific behaviour and effects of NSAIDs in the environment. Nevertheless, further research is needed to further our understanding and better appreciate the environmental risks posed by chiral NSAIDs. Therefore, future perspectives and recommendations for research in this area are outlined.

\section{Analytical strategies}

Significant progress has been made in analytical methods for enantioselective analysis of NSAIDs in environmental matrices over the past 10 years (Table 2). However, further developments are now needed to improve and ensure the quality of quantitative data, and to shorten analysis times. Although sample extraction processes, e.g. SPE, are not considered enantioselective in nature, sample storage can be. For example, enantioselective degradation of the stimulant amphetamine has been observed in surface water during storage at $4{ }^{\circ} \mathrm{C}$ for $48 \mathrm{~h}$ (Ramage et al. 2019). Many authors report the storage of water samples for NSAIDs analysis at $4{ }^{\circ} \mathrm{C}$ to mitigate any analyte losses or enantiospecific changes (Table 2). However, the suitability of this approach needs investigated for NSAIDs considering chiral inversion and degradation could take place.

A further consideration needed to ensure accurate data are collected is the method of quantitation used. A well-known issue of environmental analysis using electrospray ionization in LC-MS applications is the effect of co-extracted matrix components on analyte signal strength (Cappiello et al. 2008; Furey et al. 2013; Petrović et al. 2005). This can lead to enhancement or suppression of signal strength, which can also be enantioselective (Camacho-Muñoz and Kasprzyk-Hordern 2015, 2017; Camacho-Muñoz et al. 2016; Castrignanò et al. 2018; López-Serna et al. 2013). Such effects can be easily corrected using deuterated surrogates in the analysis process. However, several methods report the use of matrix matched calibrations without deuterated surrogates/internal standards to account for these matrix effects (Li et al. 2019; Ma et al. 2019; Wang et al. 2018; Yuan et al. 2018) (Table 2). This quantification approach needs care, particularly in monitoring studies where the composition of 
Table 5 Enantiospecific toxicity data for non-steroidal anti-inflammatory drugs

\begin{tabular}{|c|c|c|c|c|c|c|c|c|c|}
\hline Drug & $\begin{array}{l}\text { Testing } \\
\text { organism } \\
\text { group }\end{array}$ & $\begin{array}{l}\text { Testing } \\
\text { organism }\end{array}$ & Endpoint & $\begin{array}{l}\text { Toxicity } \\
\text { measure }\end{array}$ & $\begin{array}{l}\text { Exposure } \\
\text { time }\end{array}$ & $\begin{array}{l}R(-)- \\
\text { enantiomer } \\
\left(\mathrm{mg} \mathrm{L}^{-1}\right)\end{array}$ & $\begin{array}{l}S(+) \text {-enanti- } \\
\text { omer } \\
\left(\mathrm{mg} \mathrm{L}^{-1}\right)\end{array}$ & $\begin{array}{l}\text { Enan- } \\
\text { tiospecific } \\
\text { difference }^{a}\end{array}$ & References \\
\hline \multirow[t]{22}{*}{ Naproxen } & Bacteria & P. leiognathi & $\begin{array}{r}\text { Bacterial } \\
\text { toxicity }\end{array}$ & $\mathrm{EC}_{50}$ & $30 \mathrm{~min}$ & 0.75 & 0.93 & 1.2 & A \\
\hline & Algae & $\begin{array}{l}\text { P. subcapi- } \\
\text { tata }\end{array}$ & $\begin{array}{l}\text { PSII Inhibi- } \\
\text { tion }\end{array}$ & $\mathrm{EC}_{10}$ & $2 \mathrm{~h}$ & 20.1 & $>18.8$ & - & A \\
\hline & Algae & $\begin{array}{l}\text { P. subcapi- } \\
\text { tata }\end{array}$ & $\begin{array}{l}\text { PSII Inhibi- } \\
\text { tion }\end{array}$ & $\mathrm{EC}_{10}$ & $24 \mathrm{~h}$ & 19.8 & 23.8 & 1.2 & A \\
\hline & $\begin{array}{l}\text { Fish liver } \\
\text { cells }\end{array}$ & PLHC-1 & $\begin{array}{l}\text { EROD activ- } \\
\text { ity }\end{array}$ & $\mathrm{EC}_{\mathrm{IR} 1.5}$ & $6 \mathrm{~h}$ & 12.6 & 5.1 & 2.5 & A \\
\hline & $\begin{array}{l}\text { Fish liver } \\
\text { cells }\end{array}$ & PLHC-1 & Cell viability & $\mathrm{EC}_{50}$ & $48 \mathrm{~h}$ & $>18.8$ & $>13.2$ & - & A \\
\hline & Invertebrate & D. magna & Immobility & $\mathrm{EC}_{50}$ & $48 \mathrm{~h}$ & - & 85.3 & - & B \\
\hline & Invertebrate & $\begin{array}{l}\text { M. macro- } \\
\text { copa }\end{array}$ & Immobility & $\mathrm{EC}_{50}$ & $48 \mathrm{~h}$ & - & 74.1 & - & B \\
\hline & Invertebrate & D. magna & Survival & NOEC & 21 days & - & 30 & - & B \\
\hline & Invertebrate & D. magna & $\begin{array}{l}\text { Reproduc- } \\
\text { tion }\end{array}$ & NOEC & 21 days & - & 10 & - & B \\
\hline & Invertebrate & D. magna & Growth & NOEC & 21 days & - & 10 & - & B \\
\hline & Invertebrate & $\begin{array}{l}\text { M. macro- } \\
\text { сора }\end{array}$ & Survival & NOEC & 7 days & - & 30 & - & B \\
\hline & Invertebrate & $\begin{array}{l}\text { M. macro- } \\
\text { сора }\end{array}$ & $\begin{array}{l}\text { Reproduc- } \\
\text { tion }\end{array}$ & NOEC & 7 days & - & 0.3 & - & B \\
\hline & Fish & O. latipes & Survival & NOEC & 40 days & - & 0.5 & - & B \\
\hline & Fish & O. latipes & Growth & NOEC & 40 days & - & 50 & - & B \\
\hline & Fish & $\begin{array}{l}\text { D. rerio } \\
\text { (embryo) }\end{array}$ & $\begin{array}{l}\text { Immobility/ } \\
\text { Death }\end{array}$ & $\mathrm{LC}_{50}$ & $96 \mathrm{~h}$ & - & 115.2 & - & $\mathrm{C}$ \\
\hline & Fish & $\begin{array}{l}\text { D. rerio } \\
\text { (larvae) }\end{array}$ & $\begin{array}{l}\text { Immobility/ } \\
\text { Death }\end{array}$ & $\mathrm{LC}_{50}$ & $96 \mathrm{~h}$ & - & 147.6 & - & $\mathrm{C}$ \\
\hline & Fish & $\begin{array}{l}\text { D. rerio } \\
\text { (embryo) }\end{array}$ & $\begin{array}{l}\text { Malforma- } \\
\text { tion }\end{array}$ & $\mathrm{EC}_{50}$ & $96 \mathrm{~h}$ & - & 98.3 & - & $\mathrm{C}$ \\
\hline & Fish & $\begin{array}{l}\text { D. rerio } \\
\text { (larvae) }\end{array}$ & $\begin{array}{l}\text { Malforma- } \\
\text { tion }\end{array}$ & $\mathrm{EC}_{50}$ & $96 \mathrm{~h}$ & - & 149 & - & $\mathrm{C}$ \\
\hline & Invertebrate & D. magna & Immobility & $\mathrm{EC}_{50}$ & $48 \mathrm{~h}$ & - & 46.7 & - & $\mathrm{D}$ \\
\hline & Fish & C. carpio & Immobility & $\mathrm{LC}_{50}$ & $96 \mathrm{~h}$ & - & 269.2 & - & $\mathrm{D}$ \\
\hline & Algae & $\begin{array}{l}\text { S. subspica- } \\
\text { tus }\end{array}$ & Growth & $\mathrm{EC}_{50}$ & $72 \mathrm{~h}$ & - & 625.5 & - & $\mathrm{E}$ \\
\hline & Invertebrate & D. magna & Immobility & $\mathrm{EC}_{50}$ & $24-48 \mathrm{~h}$ & - & 166.3 & - & E \\
\hline \multirow[t]{5}{*}{ Flurbiprofen } & Bacteria & P. leiognathi & $\begin{array}{r}\text { Bacterial } \\
\text { toxicity }\end{array}$ & $\mathrm{EC}_{50}$ & $30 \mathrm{~min}$ & 2.13 & 1.22 & 1.7 & A \\
\hline & Algae & $\begin{array}{l}\text { P. subcapi- } \\
\text { tata }\end{array}$ & $\begin{array}{l}\text { PSII Inhibi- } \\
\text { tion }\end{array}$ & $\mathrm{EC}_{10}$ & $2 \mathrm{~h}$ & 6.92 & 9.79 & 1.4 & A \\
\hline & Algae & $\begin{array}{l}\text { P. subcapi- } \\
\quad \text { tata }\end{array}$ & $\begin{array}{l}\text { PSII Inhibi- } \\
\text { tion }\end{array}$ & $\mathrm{EC}_{10}$ & $24 \mathrm{~h}$ & 5.47 & 9.07 & 1.7 & A \\
\hline & $\begin{array}{l}\text { Fish liver } \\
\text { cells }\end{array}$ & PLHC-1 & $\begin{array}{l}\text { EROD activ- } \\
\text { ity }\end{array}$ & $\mathrm{EC}_{\mathrm{IR} 1.5}$ & $6 \mathrm{~h}$ & 8.39 & $>12.5$ & $>1.5$ & A \\
\hline & $\begin{array}{l}\text { Fish liver } \\
\text { cells }\end{array}$ & PLHC-1 & Cell viability & $\mathrm{EC}_{50}$ & $48 \mathrm{~h}$ & $>13.8$ & $>13.7$ & - & A \\
\hline \multirow[t]{3}{*}{ Ibuprofen } & Algae & $\begin{array}{l}\text { C. pyrenoi- } \\
\text { dosa }\end{array}$ & Growth & $\mathrm{IC}_{50}$ & $48 \mathrm{~h}$ & 66.7 & 65.5 & - & $\mathrm{F}$ \\
\hline & Algae & $\begin{array}{l}\text { C. pyrenoi- } \\
\text { dosa }\end{array}$ & Growth & $\mathrm{IC}_{50}$ & $72 \mathrm{~h}$ & 64.4 & 53.8 & 1.2 & $\mathrm{~F}$ \\
\hline & Algae & $\begin{array}{l}\text { C. pyrenoi- } \\
\text { dosa }\end{array}$ & Growth & $\mathrm{IC}_{50}$ & $96 \mathrm{~h}$ & 61.0 & 54.5 & 1.1 & $\mathrm{~F}$ \\
\hline
\end{tabular}


Table 5 (continued)

\begin{tabular}{|c|c|c|c|c|c|c|c|c|c|}
\hline Drug & $\begin{array}{l}\text { Testing } \\
\text { organism } \\
\text { group }\end{array}$ & $\begin{array}{l}\text { Testing } \\
\text { organism }\end{array}$ & Endpoint & $\begin{array}{l}\text { Toxicity } \\
\text { measure }\end{array}$ & $\begin{array}{l}\text { Exposure } \\
\text { time }\end{array}$ & $\begin{array}{l}R(-)- \\
\text { enantiomer } \\
\left(\mathrm{mg} \mathrm{L}^{-1}\right)\end{array}$ & $\begin{array}{l}S(+) \text {-enanti- } \\
\text { omer } \\
\left(\mathrm{mg} \mathrm{L}^{-1}\right)\end{array}$ & $\begin{array}{l}\text { Enan- } \\
\text { tiospecific } \\
\text { difference }^{a}\end{array}$ & References \\
\hline & Bacteria & P. leiognathi & $\begin{array}{l}\text { Bacterial } \\
\text { toxicity }\end{array}$ & $\mathrm{EC}_{50}$ & $30 \mathrm{~min}$ & 2.84 & 3.13 & 1.1 & A \\
\hline & Algae & $\begin{array}{l}\text { P. subcapi- } \\
\text { tata }\end{array}$ & $\begin{array}{l}\text { PSII Inhibi- } \\
\text { tion }\end{array}$ & $\mathrm{EC}_{10}$ & $2 \mathrm{~h}$ & $>18.5$ & $>29.5$ & - & A \\
\hline & Algae & $\begin{array}{l}\text { P. subcapi- } \\
\text { tata }\end{array}$ & $\begin{array}{l}\text { PSII Inhibi- } \\
\text { tion }\end{array}$ & $\mathrm{EC}_{10}$ & $24 \mathrm{~h}$ & $>18.5$ & $>29.5$ & - & A \\
\hline & $\begin{array}{l}\text { Fish liver } \\
\text { cells }\end{array}$ & PLHC-1 & $\begin{array}{l}\text { EROD activ- } \\
\text { ity }\end{array}$ & $\mathrm{EC}_{\mathrm{IR} 1.5}$ & $6 \mathrm{~h}$ & $>11.9$ & $>19.0$ & - & A \\
\hline & $\begin{array}{l}\text { Fish liver } \\
\text { cells }\end{array}$ & PLHC-1 & Cell viability & $\mathrm{EC}_{50}$ & $24 \mathrm{~h}$ & $>13.0$ & $>20.7$ & - & A \\
\hline \multirow[t]{8}{*}{ Ketoprofen } & Bacteria & P. leiognathi & $\begin{array}{r}\text { Bacterial } \\
\text { toxicity }\end{array}$ & $\mathrm{EC}_{50}$ & $30 \mathrm{~min}$ & 4.23 & 4.56 & - & A \\
\hline & Algae & $\begin{array}{l}\text { P. subcapi- } \\
\quad \text { tata }\end{array}$ & $\begin{array}{l}\text { PSII Inhibi- } \\
\text { tion }\end{array}$ & $\mathrm{EC}_{10}$ & $2 \mathrm{~h}$ & $>16.7$ & $>42.3$ & - & A \\
\hline & Algae & $\begin{array}{l}\text { P. subcapi- } \\
\quad \text { tata }\end{array}$ & $\begin{array}{l}\text { PSII Inhibi- } \\
\text { tion }\end{array}$ & $\mathrm{EC}_{10}$ & $24 \mathrm{~h}$ & $>16.7$ & $>42.3$ & - & A \\
\hline & $\begin{array}{l}\text { Fish liver } \\
\text { cells }\end{array}$ & PLHC-1 & $\begin{array}{l}\text { EROD activ- } \\
\text { ity }\end{array}$ & $\mathrm{EC}_{\mathrm{IR} 1.5}$ & $6 \mathrm{~h}$ & 4.34 & $>27.2$ & $>6.3$ & A \\
\hline & $\begin{array}{l}\text { Fish liver } \\
\text { cells }\end{array}$ & PLHC-1 & Cell viability & $\mathrm{EC}_{50}$ & $24 \mathrm{~h}$ & $>11.7$ & $>29.6$ & - & A \\
\hline & Algae & $\begin{array}{l}\text { P. subcapi- } \\
\quad \text { tata }\end{array}$ & Growth & $\mathrm{EC}_{50}$ & $96 \mathrm{~h}$ & - & 0.066 & - & G \\
\hline & Algae & $\begin{array}{l}\text { P. subcapi- } \\
\quad \text { tata }\end{array}$ & Growth & NOEC & $96 \mathrm{~h}$ & - & 0.004 & - & G \\
\hline & Algae & $\begin{array}{l}\text { P. subcapi- } \\
\text { tata }\end{array}$ & Growth & LOEC & $96 \mathrm{~h}$ & - & 0.008 & - & $\mathrm{G}$ \\
\hline
\end{tabular}

Key: $\mathrm{EC}_{10}$, effective concentration, $10 \% ; \mathrm{EC}_{50}$, half maximal effective concentration; $\mathrm{EC}_{\mathrm{IR} 1.5}$, concentration that induces an induction ratio of 1.5; EROD, ethoxyresorufin-O-deethylase; $\mathrm{LC}_{50}$, lethal concentration, 50\%; LOEC, lowest observed effect concentration; NOEC, no observed effect concentration; PSII, photosystem II

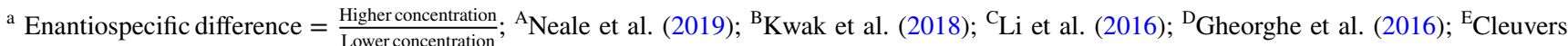

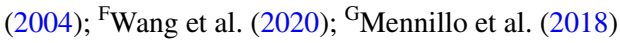

collected samples can change between samples. For example, the signal suppression of the pesticide aldicarb for five different apple varieties varied by $42 \%$ (Kruve et al. 2008).

Considering LC is the preferred method of analysis and is likely to continue being so, it is important to note that existing methods use enantioselective columns with $5 \mu \mathrm{m}$ stationary phase particle sizes (Table 2). Such columns are restricted to use in high-performance liquid chromatography operation. Commercially available columns particularly for the polysaccharide phases are now available with sub- $2 \mu \mathrm{m}$ particle sizes. The ability to operate at ultra-performance liquid chromatography pressures facilitates higher plate numbers, improved resolution and shorter analysis times.

\section{Occurrence and fate studies}

Several studies have reported enantioselective changes to NSAIDs during wastewater treatment and in the environment
(Tables 3, 4). Both chiral inversion and enantioselective degradation have been demonstrated (Bertin et al. 2020; Hashim and Khan 2011; Nguyen et al. 2017; Suzuki et al. 2014). These studies have shown that it is essential to conduct microcosm studies using individual enantiomers over the racemate where possible to better evaluate enantioselective fate processes. However, studies evidencing the mechanisms of inversion and degradation in an environmental context are lacking. This is challenging for complex environmental compartments including those encountered in engineered systems such as wastewater treatment plants. Nevertheless, studies need to be undertaken on individual microbial species found in wastewater treatment plants to better our understanding of NSAIDs fate during wastewater treatment. This will also help explain the differences in enantioselectivity observed between the same process types, as well as different processes (Tables 3, 4). 
The majority of enantioselective occurrence and fate investigations on NSAIDs are focused on aqueous environmental matrices (Tables 3, 4). Chiral NSAIDs have been reported in particulate matrices such as treated and untreated sludge, soils and sediments, albeit not at the enantiomeric level (Albero et al. 2014; Kumirska et al. 2015; Martín et al. 2012; Radjenović et al. 2009a). Although there is some enantiomeric information for ibuprofen and naproxen in soils (Bertin et al. 2020), little enantiospecific data exist for sediments or sludges. Yuan et al. (2018) reported enantiomeric data for ibuprofen in sediment and sludge. However, the order of enantiomer elution is unknown and chromatographic peaks could not be assigned $S(+)$-ibuprofen and $R(-)$-ibuprofen. Nevertheless, the data showed ibuprofen to be present in sludge in non-racemic form (Yuan et al. 2018). Therefore, further studies on the enantioselective occurrence and behaviour of NSAIDs in particulate environmental matrices are recommended. Methods which can support such studies are already available or can be adapted to do so (Bertin et al. 2020; Yuan et al. 2018).

Investigations on the enantiospecific occurrence and fate of metabolites during wastewater treatment and in the environment can help better understand the fate of the parent compounds. Several methods exist which facilitate enantioseparation of metabolites including carboxyibuprofen, 2-hydroxyibuprofen and dihydroketoprofen (CamachoMuñoz and Kasprzyk-Hordern 2015; 2017; CamachoMuñoz et al. 2016) (Table 2). In such methods, the order of enantiomer elution is not known. Without the use of optical rotation detection to determine enantiomer elution order, the commercial availability of enantiomerically pure reference standards is essential. Such standards are also needed to conduct single enantiomer microcosm studies. However, at present such reference standards are not available for most NSAID metabolites expect for $S(+)$-O-desmethylnaproxen and $R(-)$-O-desmethylnaproxen. The availability of enantiopure metabolite standards would also facilitate enantiospecific toxicity studies. Many metabolites are themselves pharmacologically active and have been reported in river waters (Paíga et al. 2016; Zha et al. 2017; Zojaji et al. 2019).

\section{Ecotoxicity studies}

Existing data show that although enantiospecific differences exist, they were less than one order or magnitude (Neale et al. 2019). Nevertheless, there is need for additional enantiospecific investigation for a greater range of organisms and endpoints considering the enantiomer enrichment observed in the environment (Table 3). Toxicity testing also needs to investigate chronic exposure as the majority of research undertaken to date has focussed on acute effects (Table 5). It is recommended that these are undertaken at environmentally relevant concentrations as the majority of acute exposures use enantiomer concentrations considerably greater than those encountered in the environment (Table 5). This will improve the accuracy of environmental risk assessments of chiral NSAIDs and establish the significance of considering stereochemistry for the environmental effects of such compounds. Metabolomic approaches are also beneficial to provide information on organism function and health at the molecular level (Bundy et al. 2008). This will provide additional information which would otherwise be missed by traditional bioassays and endpoints. Once a greater body of knowledge is available on the enantiospecific effects of chiral NSAIDs and their modes of action, mixture effects can be considered.

It is suggested that effect studies are supported with appropriate enantioselective analysis to report actual concentrations over nominal concentrations in exposure media over the duration of studies. This analysis is important to undertake throughout the exposure period. Mennillo et al. (2018) reported no change to the concentration of $S(+)$-ketoprofen in $P$. subcapitata test media after $72 \mathrm{~h}$. Zhang et al. (2020) also reported drug stability during exposure of $D$. rerio with ibuprofen enantiomers. Although they confirmed the 'total' ibuprofen concentration did not vary substantially, enantioselective analysis was not used to confirm the enantiomeric composition and confirm inversion did not take place. On the other hand, the requirement to confirm drug enantiomeric composition is less critical for studies conducted on cells over whole organisms. For example, Neale et al. (2019) who undertook toxicity assays using fish cells stated that any inversion that takes place is part of the cell's response to the enantiomer and is still a relevant measure of its biological activity.

\section{Conclusion}

Investigating the stereochemistry of NSAIDs in wastewaters and the environment poses several challenges. This review demonstrates that considerable progress has been made in this research area. However, to fully assess the environmental risk posed by NSAIDs at the enantiomeric level, further work is needed as outlined in this review, e.g. fate studies using enantiopure standards, investigation of metabolites and further enantiospecific toxicity studies. Once the risk is established, appropriate mitigation steps can then be undertaken, if needed. The essential use of NSAIDs by growing and aging populations is unlikely to result in any reduction of their entry into wastewater in the future. However, modifying existing wastewater treatment plant operation to target the removal of specific enantiomers is an opportunity to reduce any identified risks. This could be achievable whereby the microbial species that preferentially degrade or invert those more toxic enantiomers are known, and wastewater treatment plants can be operated to facilitate 
their prevalence. For example, the microbial community in suspended biomass systems changes with sludge age which can be manipulated through process operation (Pala-Ozkok et al. 2013).

Open Access This article is licensed under a Creative Commons Attribution 4.0 International License, which permits use, sharing, adaptation, distribution and reproduction in any medium or format, as long as you give appropriate credit to the original author(s) and the source, provide a link to the Creative Commons licence, and indicate if changes were made. The images or other third party material in this article are included in the article's Creative Commons licence, unless indicated otherwise in a credit line to the material. If material is not included in the article's Creative Commons licence and your intended use is not permitted by statutory regulation or exceeds the permitted use, you will need to obtain permission directly from the copyright holder. To view a copy of this licence, visit http://creativecommons.org/licenses/by/4.0/.

\section{References}

Albero B, Sánchez-Brunete C, Miguel E, Aznar R, Tadeo JL (2014) Determination of selected pharmaceutical compounds in biosolids by supported liquid extraction and gas chromatography-tandem mass spectrometry. J Chromatogr A 1336:52-58. https://doi. org/10.1016/j.chroma.2014.02.020

Bagnall JP, Evans SE, Wort MT, Lubben AT, Kasprzyk-Hordern B (2012) Using chiral liquid chromatography quadrupole time-offlight mass spectrometry for the analysis of pharmaceuticals and illicit drugs in surface and wastewater at the enantiomeric level. J Chromatogr A 1249:115-129. https://doi.org/10.1016/j.chrom a.2012.06.012

Barnett J, Chow J, Ives D, Chiou M, Mackenzie R, Osen E, Nguyen B, Tsing S, Bach C, Freire J, Chan H, Sigal E, Ramesha C (1994) Purification, characterization and selective inhibition of human prostaglandin $\mathrm{G} / \mathrm{H}$ synthase 1 and 2 expressed in the baculovirus system. Biochim Biophys 1209(1):130-139. https://doi. org/10.1016/0167-4838(94)90148-1

Bao X, Snurr RQ, Broadbelt LJ (2013) Insights into the complexity of chiral recognition by a three-point model. Microporous Mesoporous Mater 172:44-50. https://doi.org/10.1016/j.micro meso.2013.01.014

Berthod A (2006) Chiral recognition mechanisms. Anal Chem 78(7):2093-2099. https://doi.org/10.1021/ac0693823

Bertin S, Yates K, Petrie B (2020) Enantiospecific behaviour of chiral drugs in soil. Environ Pollut 262:114364. https://doi. org/10.1016/j.envpol.2020.114364

Biel-Maeso M, Corada-Fernández C, Lara-Martín PA (2018) Monitoring the occurrence of pharmaceuticals in soils irrigated with reclaimed wastewater. Environ Pollut 235:312-321. https://doi. org/10.1016/j.envpol.2017.12.085

Borges KB, de Oliveira ARM, Barth T, Jabor VAP, Pupo MT, Bonato PS (2011) LC-MS-MS determination of ibuprofen, 2-hydroxyibuprofen enantiomers, and carboxyibuprofen stereoisomers for application in biotransformation studies employing endophytic fungi. Anal Bioanal Chem 399(2):915-925. https://doi. org/10.1007/s00216-010-4329-9

Boyacı E, Rodríguez-Lafuente Á, Gorynski K, Mirnaghi F, Souza-Silva ÉA, Hein D, Pawliszyn J (2015) Sample preparation with solid phase microextraction and exhaustive extraction approaches: Comparison for challenging cases. Anal Chim Acta 873:14-30. https://doi.org/10.1016/j.aca.2014.12.051
Brune K, Patrignani P (2015) New insights into the use of currently available non-steroidal anti-inflammatory drugs [Review]. J Pain Res 8:105-118. https://doi.org/10.2147/JPR.S75160

Buchberger WW (2011) Current approaches to trace analysis of pharmaceuticals and personal care products in the environment. J Chromatogr A 1218(4):603-618. https://doi.org/10.1016/j.chrom a.2010.10.040

Bundy JG, Davey MP, Viant MR (2008) Environmental metabolomics: a critical review and future perspectives. Metabolomics 5(1):3. https://doi.org/10.1007/s11306-008-0152-0

Buser H-R, Poiger T, Müller MD (1999) Occurrence and environmental behavior of the chiral pharmaceutical drug ibuprofen in surface waters and in wastewater. Environ Sci Technol 33(15):2529 2535. https://doi.org/10.1021/es981014w

Caballo C, Sicilia MD, Rubio S (2015a) Enantioselective determination of representative profens in wastewater by a single-step sample treatment and chiral liquid chromatography-tandem mass spectrometry. Talanta 134:325-332. https://doi.org/10.1016/j.talan ta.2014.11.016

Caballo C, Sicilia MD, Rubio S (2015b) Enantioselective analysis of non-steroidal anti-inflammatory drugs in freshwater fish based on microextraction with a supramolecular liquid and chiral liquid chromatography-tandem mass spectrometry. Anal Bioanal Chem 407(16):4721-4731. https://doi.org/10.1007/s00216-015-8675-5

Camacho-Muñoz D, Kasprzyk-Hordern B (2015) Multi-residue enantiomeric analysis of human and veterinary pharmaceuticals and their metabolites in environmental samples by chiral liquid chromatography coupled with tandem mass spectrometry detection. Anal Bioanal Chem 407(30):9085-9104. https://doi.org/10.1007/ s00216-015-9075-6

Camacho-Muñoz D, Kasprzyk-Hordern B (2017) Simultaneous enantiomeric analysis of pharmacologically active compounds in environmental samples by chiral LC-MS/MS with a macrocyclic antibiotic stationary phase. J Mass Spectrom 52(2):94-108. https ://doi.org/10.1002/jms.3904

Camacho-Muñoz D, Kasprzyk-Hordern B, Thomas KV (2016) Enantioselective simultaneous analysis of selected pharmaceuticals in environmental samples by ultrahigh performance supercritical fluid based chromatography tandem mass spectrometry. Anal Chim Acta 934:239-251. https://doi.org/10.1016/j. aca.2016.05.051

Camacho-Muñoz D, Martín J, Santos JL, Aparicio I, Alonso E (2012) Effectiveness of conventional and low-cost wastewater treatments in the removal of pharmaceutically active compounds. Water Air Soil Pollut 223(5):2611-2621. https://doi.org/10.1007/s1127 0-011-1053-9

Camacho-Muñoz D, Martín J, Santos JL, Aparicio I, Alonso E (2014) Concentration evolution of pharmaceutically active compounds in raw urban and industrial wastewater. Chemosphere 111:70-79. https://doi.org/10.1016/j.chemosphere.2014.03.043

Camacho-Muñoz D, Petrie B, Lopardo L, Proctor K, Rice J, Youdan J, Barden R, Kasprzyk-Hordern B (2019) Stereoisomeric profiling of chiral pharmaceutically active compounds in wastewaters and the receiving environment-a catchment-scale and a laboratory study. Environ Int 127:558-572. https://doi.org/10.1016/j.envin t.2019.03.050

Cappiello A, Famiglini G, Palma P, Pierini E, Termopoli V, Trufelli $\mathrm{H}$ (2008) Overcoming matrix effects in liquid chromatographymass spectrometry. Anal Chem 80(23):9343-9348. https://doi. org/10.1021/ac8018312

Carabaza A, Cabré F, Rotllan E, Gómez M, Gutiérrez M, García ML, Mauleón D (1996) Stereoselective inhibition of inducible cyclooxygenase by chiral nonsteroidal antiinflammatory drugs. J Clin Pharmacol 36(6):505-512. https://doi. org/10.1002/j.1552-4604.1996.tb05040.x 
Castrignanò E, Kannan AM, Feil EJ, Kasprzyk-Hordern B (2018) Enantioselective fractionation of fluoroquinolones in the aqueous environment using chiral liquid chromatography coupled with tandem mass spectrometry. Chemosphere 206:376-386. https:// doi.org/10.1016/j.chemosphere.2018.05.005

Castrignanò E, Mardal M, Rydevik A, Miserez B, Ramsey J, Shine T, Pantoș GD, Meyer MR, Kasprzyk-Hordern B (2017) A new approach towards biomarker selection in estimation of human exposure to chiral chemicals: a case study of mephedrone. Sci Rep 7(1):13009. https://doi.org/10.1038/s41598-017-12581-3

Choi PM, Li J, Gao J, O’Brien JW, Thomas KV, Thai PK, Jiang G, Mueller JF (2020) Considerations for assessing stability of wastewater-based epidemiology biomarkers using biofilm-free and sewer reactor tests. Sci Total Environ 709:136228. https:// doi.org/10.1016/j.scitotenv.2019.136228

Cizmas L, Sharma VK, Gray CM, McDonald TJ (2015) Pharmaceuticals and personal care products in waters: occurrence, toxicity, and risk. Environ Chem Lett 13:381-394. https://doi. org/10.1007/s10311-015-0524-4

Cleuvers M (2004) Mixture toxicity of the anti-inflammatory drugs diclofenac, ibuprofen, naproxen, and acetylsalicylic acid. Ecotoxicol Environ Saf 59(3):309-315. https://doi.org/10.1016/ S0147-6513(03)00141-6

Coelho MM, Lado Ribeiro AR, Sousa JCG, Ribeiro C, Fernandes C, Silva AMT, Tiritan ME (2019) Dual enantioselective LC-MS/ MS method to analyse chiral drugs in surface water: Monitoring in Douro River estuary. J Pharm Biomed Anal 170:89-101. https ://doi.org/10.1016/j.jpba.2019.03.032

Drugbank. https://www.drugbank.ca/. Accessed 2 June 2020

Eaglesham A, Scott A, Petrie B (2020) Multi-residue enantioselective analysis of chiral drugs in freshwater sediments. Environ Chem Lett. https://doi.org/10.1007/s10311-020-01041-6

Escuder-Gilabert L, Martín-Biosca Y, Perez-Baeza M, Sagrado S, Medina-Hernández MJ (2018) Direct chromatographic study of the enantioselective biodegradation of ibuprofen and ketoprofen by an activated sludge. J Chromatogr A 1568:140-148. https:// doi.org/10.1016/j.chroma.2018.07.034

Evans AM (2001) Comparative pharmacology of S(+)-ibuprofen and (RS)-ibuprofen. Clin Rheumatol 20(1):9-14. https://doi. org/10.1007/BF03342662

Evans SE, Davies P, Lubben A, Kasprzyk-Hordern B (2015) Determination of chiral pharmaceuticals and illicit drugs in wastewater and sludge using microwave assisted extraction, solid-phase extraction and chiral liquid chromatography coupled with tandem mass spectrometry. Anal Chim Acta 882:112-126. https://doi. org/10.1016/j.aca.2015.03.039

Evans SE, Kasprzyk-Hordern B (2014) Applications of chiral chromatography coupled with mass spectrometry in the analysis of chiral pharmaceuticals in the environment. TrAC Trends Environ Anal Chem 1:e34-e51. https://doi.org/10.1016/j.teac.2013.11.005

Evans AM, Nation RL, Sansom LN, Bochner F, Somogyi AA (1991) Effect of racemic ibuprofen dose on the magnitude and duration of platelet cyclo-oxygenase inhibition: relationship between inhibition of thromboxane production and the plasma unbound concentration of S(+)-ibuprofen. Br J Clin Pharmacol 31(2):131138. https://doi.org/10.1111/j.1365-2125.1991.tb05500.x

Farré M, Kantiani L, Petrovic M, Pérez S, Barceló D (2012) Achievements and future trends in the analysis of emerging organic contaminants in environmental samples by mass spectrometry and bioanalytical techniques. J Chromatogr A 1259:86-99. https:// doi.org/10.1016/j.chroma.2012.07.024

Fent K (2001) Fish cell lines as versatile tools in ecotoxicology: assessment of cytotoxicity, cytochrome P4501A induction potential and estrogenic activity of chemicals and environmental samples. Toxicol In Vitro 15(4):477-488. https://doi.org/10.1016/S0887 $-2333(01) 00053-4$
Fernandes C, Tiritan ME, Pinto M (2013) Small Molecules as chromatographic tools for HPLC enantiomeric resolution: Pirkle-type chiral stationary phases evolution. Chromatographia 76(15):871897. https://doi.org/10.1007/s10337-013-2469-8

Fono LJ, Sedlak DL (2005) Use of the chiral pharmaceutical propranolol to identify sewage discharges into surface waters. Environ Sci Technol 39(23):9244-9252. https://doi.org/10.1021/es047965t

Furey A, Moriarty M, Bane V, Kinsella B, Lehane M (2013) Ion suppression; A critical review on causes, evaluation, prevention and applications. Talanta 115:104-122. https://doi.org/10.1016/j. talanta.2013.03.048

Gao J, Li J, Jiang G, Shypanski AH, Nieradzik LM, Yuan Z, Mueller JF, Ort C, Thai PK (2019) Systematic evaluation of biomarker stability in pilot scale sewer pipes. Water Res 151:447-455. https ://doi.org/10.1016/j.watres.2018.12.032

Gardner M, Jones V, Comber S, Scrimshaw MD, Coello-Garcia T, Cartmell E, Lester J, Ellor B (2013) Performance of UK wastewater treatment works with respect to trace contaminants. Sci Total Environ 456-457:359-369. https://doi.org/10.1016/j.scito tenv.2013.03.088

Garzotti M, Hamdan M (2002) Supercritical fluid chromatography coupled to electrospray mass spectrometry: a powerful tool for the analysis of chiral mixtures. J Chromatogr B 770(1):53-61. https ://doi.org/10.1016/S1570-0232(01)00582-7

Gheorghe S, Petre J, Lucaciu I, Stoica C, Nita-Lazar M (2016) Risk screening of pharmaceutical compounds in Romanian aquatic environment. Environ Monit Assess 188(6):379. https://doi. org/10.1007/s10661-016-5375-3

Ghezzi P (1998) Differential contribution of $r$ and s isomers in ketoprofen anti-inflammatory activity: role of cytokine modulation. J Pharmacol Exp Ther 287(3):969-974

Gibson R, Durán-Álvarez JC, Estrada KL, Chávez A, Jiménez Cisneros B (2010) Accumulation and leaching potential of some pharmaceuticals and potential endocrine disruptors in soils irrigated with wastewater in the Tula Valley, Mexico. Chemosphere 81(11):1437-1445. https://doi.org/10.1016/j.chemospher e.2010.09.006

Haginaka J (2001) Protein-based chiral stationary phases for highperformance liquid chromatography enantioseparations. J Chromatogr A 906(1):253-273. https://doi.org/10.1016/S0021 $-9673(00) 00504-5$

Hamman C, Wong M, Hayes M, Gibbons P (2011) A high throughput approach to purifying chiral molecules using $3 \mu \mathrm{m}$ analytical chiral stationary phases via supercritical fluid chromatography. J Chromatogr A 1218(22):3529-3536. https://doi.org/10.1016/j. chroma.2011.03.066

Hao H, Wang G, Sun J, Ding Z, Wu X, Roberts M (2005) Unidirectional inversion of ibuprofen in Caco- 2 cells: developing a suitable model for presystemic chiral inversion study. Biol Pharm Bull 28(4):682-687. https://doi.org/10.1248/bpb.28.682

Harrington PJ, Lodewijk E (1997) Twenty years of naproxen technology. Org Process Res Dev 1(1):72-76. https://doi.org/10.1021/ op960009e

Hashim NH, Khan SJ (2011) Enantioselective analysis of ibuprofen, ketoprofen and naproxen in wastewater and environmental water samples. J Chromatogr A 1218(29):4746-4754. https://doi. org/10.1016/j.chroma.2011.05.046

Hashim NH, Nghiem LD, Stuetz RM, Khan SJ (2011) Enantiospecific fate of ibuprofen, ketoprofen and naproxen in a laboratory-scale membrane bioreactor. Water Res 45(18):6249-6258. https://doi. org/10.1016/j.watres.2011.09.020

Hashim NH, Stuetz RM, Khan SJ (2013) Enantiomeric fraction determination of 2-arylpropionic acids in a package plant membrane bioreactor. Chirality 25(5):301-307. https://doi.org/10.1002/ chir.22151 
Hermansson J (1983) Direct liquid chromatographic resolution of racemic drugs using $\alpha 1$-acid glycoprotein as the chiral stationary phase. J Chromatogr A 269:71-80. https://doi.org/10.1016/ S0021-9673(01)90787-3

Hermansson J, Hermansson I (1994) Dynamic modification of the chiral bonding properties of a CHIRAL-AGP column by organic and inorganic additives: Separation of enantiomers of antiinflammatory drugs. J Chromatogr A 666(1):181-191. https:// doi.org/10.1016/0021-9673(94)80380-3

Ilisz I, Aranyi A, Pataj Z, Péter A (2013) Enantioseparations by highperformance liquid chromatography using macrocyclic glycopeptide-based chiral stationary phases: an overview. In: Scriba GKE (ed) Chiral separations: methods and protocols. Humana Press, Totowa, pp 137-163. https://doi.org/10.1007/978-1-62703 $-263-6 \_8$

Kasprzyk-Hordern B (2010) Pharmacologically active compounds in the environment and their chirality. Chem Soc Rev 39(11):44664503. https://doi.org/10.1039/C000408C

Kasprzyk-Hordern B, Baker DR (2012) Enantiomeric profiling of chiral drugs in wastewater and receiving waters. Environ Sci Technol 46(3):1681-1691. https://doi.org/10.1021/es203113y

Kasprzyk-Hordern B, Dinsdale RM, Guwy AJ (2009) The removal of pharmaceuticals, personal care products, endocrine disruptors and illicit drugs during wastewater treatment and its impact on the quality of receiving waters. Water Res 43(2):363-380. https ://doi.org/10.1016/j.watres.2008.10.047

Kato D-I, Miyamoto K, Ohta H (2004) Microbial deracemization of $\alpha$-substituted carboxylic acids: control of the reaction path. Tetrahedron Asymmetry 15(18):2965-2973. https://doi.org/10.1016/j. tetasy.2004.06.049

Kato DI, Mitsuda S, Ohta H (2003) Microbial deracemization of $\alpha$-substituted carboxylic acids: substrate specificity and mechanistic investigation. J Org Chem 68(19):7234-7242. https://doi. org/10.1021/jo034253x

Khan SJ, Wang L, Hashim NH, Mcdonald JA (2014) Distinct enantiomeric signals of ibuprofen and naproxen in treated wastewater and sewer overflow. Chirality 26(11):739-746. https://doi. org/10.1002/chir.22258

Kruve A, Künnapas A, Herodes K, Leito I (2008) Matrix effects in pesticide multi-residue analysis by liquid chromatographymass spectrometry. J Chromatogr A 1187(1):58-66. https://doi. org/10.1016/j.chroma.2008.01.077

Kumirska J, Migowska N, Caban M, Łukaszewicz P, Stepnowski $P$ (2015) Simultaneous determination of non-steroidal antiinflammatory drugs and oestrogenic hormones in environmental solid samples. Sci Total Environ 508:498-505. https://doi. org/10.1016/j.scitotenv.2014.12.020

Kwak K, Ji K, Kho Y, Kim P, Lee J, Ryu J, Choi K (2018) Chronic toxicity and endocrine disruption of naproxen in freshwater waterfleas and fish, and steroidogenic alteration using H295R cell assay. Chemosphere 204:156-162. https://doi.org/10.1016/j. chemosphere.2018.04.035

Laneuville O, Breuer DK, Dewitt DL, Hla T, Funk CD, Smith WL (1994) Differential inhibition of human prostaglandin endoperoxide $\mathrm{H}$ synthases- 1 and -2 by nonsteroidal anti-inflammatory drugs. J Pharmacol Exp Ther 271(2):927-934

Larsson E, al-Hamimi S, Jönsson JA (2014) Behaviour of nonsteroidal anti-inflammatory drugs and eight of their metabolites during wastewater treatment studied by hollow fibre liquid phase microextraction and liquid chromatography mass spectrometry. Sci Total Environ 485-486:300-308. https://doi.org/10.1016/j. scitotenv.2014.03.055

Li J, Gao J, Thai PK, Shypanski A, Nieradzik L, Mueller JF, Yuan Z, Jiang G (2019) Experimental investigation and modeling of the transformation of illicit drugs in a pilot-scale sewer system.
Environ Sci Technol 53(8):4556-4565. https://doi.org/10.1021/ acs.est.8b06169

Li M, Liang X, Guo X, Di X, Jiang Z (2020) Enantiomeric separation and enantioselective determination of some representive non-steroidal anti-inflammatory drug enantiomers in fish tissues by using chiral liquid chromatography coupled with tandem mass spectrometry. Microchem J 153:104511. https://doi. org/10.1016/j.microc.2019.104511

Li Q, Wang P, Chen L, Gao H, Wu L (2016) Acute toxicity and histopathological effects of naproxen in zebrafish (Danio rerio) early life stages. Environ Sci Pollut Res 23(18):18832-18841. https:// doi.org/10.1007/s11356-016-7092-4

Llompart M, Celeiro M, Dagnac T (2019) Microwave-assisted extraction of pharmaceuticals, personal care products and industrial contaminants in the environment. TrAC Trends Anal Chem 116:136-150. https://doi.org/10.1016/j.trac.2019.04.029

López-Serna R, Kasprzyk-Hordern B, Petrović M, Barceló D (2013) Multi-residue enantiomeric analysis of pharmaceuticals and their active metabolites in the Guadalquivir River basin (South Spain) by chiral liquid chromatography coupled with tandem mass spectrometry. Anal Bioanal Chem 405(18):5859-5873. https://doi. org/10.1007/s00216-013-6900-7

Ma R, Qu H, Wang B, Wang F, Yu Y, Yu G (2019) Simultaneous enantiomeric analysis of non-steroidal anti-inflammatory drugs in environment by chiral LC-MS/MS: a pilot study in Beijing, China. Ecotoxicol Environ Saf 174:83-91. https://doi. org/10.1016/j.ecoenv.2019.01.122

Madikizela LM, Tavengwa NT, Chimuka L (2018) Applications of molecularly imprinted polymers for solid-phase extraction of non-steroidal anti-inflammatory drugs and analgesics from environmental waters and biological samples. J Pharm Biomed Anal 147:624-633. https://doi.org/10.1016/j.jpba.2017.04.010

Martín J, Camacho-Muñoz MD, Santos JL, Aparicio I, Alonso E (2012) Distribution and temporal evolution of pharmaceutically active compounds alongside sewage sludge treatment. Risk assessment of sludge application onto soils. J Environ Manag 102:18-25. https://doi.org/10.1016/j.jenvman.2012.02.020

Matamoros V, Hijosa M, Bayona JM (2009) Assessment of the pharmaceutical active compounds removal in wastewater treatment systems at enantiomeric level. Ibuprofen and naproxen. Chemosphere 75(2):200-205. https://doi.org/10.1016/j.chemospher e.2008.12.008

Mennillo E, Arukwe A, Monni G, Meucci V, Intorre L, Pretti C (2018) Ecotoxicological properties of ketoprofen and the $\mathrm{S}(+)$-enantiomer (dexketoprofen): bioassays in freshwater model species and biomarkers in fish PLHC-1 cell line. Environ Toxicol Chem 37(1):201-212. https://doi.org/10.1002/etc.3943

Michishita T, Franco P, Zhang T (2010) New approaches of LC-MS compatible method development on $\alpha 1$-acid glycoprotein-based stationary phase for resolution of enantiomers by HPLC. J Sep Sci 33(23-24):3627-3637. https://doi.org/10.1002/jssc.20100 0627

Mitsukura K, Yoshida T, Nagasawa T (2002) Synthesis of (R)-2-phenylpropanoic acid from its racemate through an isomeraseinvolving reaction by Nocardia diaphanozonaria. Biotech Lett 24(19):1615-1621. https://doi.org/10.1023/A:1020353631566

Nakada N, Tanishima T, Shinohara H, Kiri K, Takada H (2006) Pharmaceutical chemicals and endocrine disrupters in municipal wastewater in Tokyo and their removal during activated sludge treatment. Water Res 40(17):3297-3303. https://doi. org/10.1016/j.watres.2006.06.039

Neale PA, Branch A, Khan SJ, Leusch FDL (2019) Evaluating the enantiospecific differences of non-steroidal anti-inflammatory drugs (NSAIDs) using an ecotoxicity bioassay test battery. Sci Total Environ 694:133659. https://doi.org/10.1016/j.scito tenv.2019.133659 
Nguyen LN, Hai FI, McDonald JA, Khan SJ, Price WE, Nghiem LD (2017) Continuous transformation of chiral pharmaceuticals in enzymatic membrane bioreactors for advanced wastewater treatment. Water Sci Technol 76(7):1816-1826. https://doi. org/10.2166/wst.2017.331

Onesios KM, Yu JT, Bouwer EJ (2009) Biodegradation and removal of pharmaceuticals and personal care products in treatment systems: a review. Biodegradation 20(4):441-466. https://doi.org/10.1007/ s10532-008-9237-8

Paíga P, Santos LHMLM, Ramos S, Jorge S, Silva JG, Delerue-Matos C (2016) Presence of pharmaceuticals in the Lis river (Portugal): sources, fate and seasonal variation. Sci Total Environ 573:164177. https://doi.org/10.1016/j.scitotenv.2016.08.089

Pala-Ozkok I, Rehman A, Kor-Bicakci G, Ural A, Schihalbel MB, Ubay-Cokgor E, Jonas D, Orhon D (2013) Effect of sludge age on population dynamics and acetate utilization kinetics under aerobic conditions. Biores Technol 143:68-75. https://doi. org/10.1016/j.biortech.2013.05.095

Palomer A, Pérez JJ, Navea S, Llorens O, Pascual J, García L, Mauleón D (2000) Modeling cyclooxygenase inhibition. Implication of active site hydration on the selectivity of ketoprofen analogues. J Med Chem 43(11):2280-2284. https://doi.org/10.1021/jm990621

Patel M, Kumar R, Kishor K, Mlsna T, Pittman CU, Mohan D (2019) Pharmaceuticals of emerging concern in aquatic systems: chemistry, occurrence, effects, and removal methods. Chem Rev 119(6):3510-3673. https://doi.org/10.1021/acs.chemrev.8b00299

Petrie B, Barden R, Kasprzyk-Hordern B (2015) A review on emerging contaminants in wastewaters and the environment: current knowledge, understudied areas and recommendations for future monitoring. Water Res 72:3-27. https://doi.org/10.1016/j.watre s.2014.08.053

Petrie B, McAdam EJ, Lester JN, Cartmell E (2014) Obtaining process mass balances of pharmaceuticals and triclosan to determine their fate during wastewater treatment. Sci Total Environ 497498:553-560. https://doi.org/10.1016/j.scitotenv.2014.08.003

Petrie B, Mrazova J, Kasprzyk-Hordern B, Yates K (2018) Multi-residue analysis of chiral and achiral trace organic contaminants in soil by accelerated solvent extraction and enantioselective liquid chromatography tandem-mass spectrometry. J Chromatogr A 1572:62-71. https://doi.org/10.1016/j.chroma.2018.08.034

Petrović M, Hernando MD, Díaz-Cruz MS, Barceló D (2005) Liquid chromatography-tandem mass spectrometry for the analysis of pharmaceutical residues in environmental samples: a review. $\mathbf{J}$ Chromatogr A 1067(1):1-14. https://doi.org/10.1016/j.chrom a.2004.10.110

Pirkle WH, Pochapsky TC (1989) Considerations of chiral recognition relevant to the liquid chromatography separation of enantiomers. Chem Rev 89(2):347-362. https://doi.org/10.1021/cr00092a006

Płotka-Wasylka J, Szczepańska N, de la Guardia M, Namieśnik J (2016) Modern trends in solid phase extraction: new sorbent media. TrAC Trends Anal Chem 77:23-43. https://doi.org/10.1016/j. trac.2015.10.010

Płotka JM, Biziuk M, Morrison C, Namieśnik J (2014) Pharmaceutical and forensic drug applications of chiral supercritical fluid chromatography. TrAC Trends Anal Chem 56:74-89. https://doi. org/10.1016/j.trac.2013.12.012

Poggi JC, Barissa GR, Donadi EA, Foss MC, de Queiróz Cunha F, Lanchote VL, dos Reis ML (2006) Pharmacodynamics, chiral pharmacokinetics, and pharmacokinetic-pharmacodynamic modeling of fenoprofen in patients with diabetes mellitus. J Clin Pharmacol 46(11):1328-1336. https://doi.org/10.1177/0091270006293072

Radjenović J, Jelić A, Petrović M, Barceló D (2009a) Determination of pharmaceuticals in sewage sludge by pressurized liquid extraction (PLE) coupled to liquid chromatography-tandem mass spectrometry (LC-MS/MS). Anal Bioanal Chem 393(6):1685-1695. https://doi.org/10.1007/s00216-009-2604-4
Radjenović J, Petrović M, Barceló D (2009b) Fate and distribution of pharmaceuticals in wastewater and sewage sludge of the conventional activated sludge (CAS) and advanced membrane bioreactor (MBR) treatment. Water Res 43(3):831-841. https://doi. org/10.1016/j.watres.2008.11.043

Ramage S, Camacho-Muñoz D, Petrie B (2019) Enantioselective LCMS/MS for anthropogenic markers of septic tank discharge. Chemosphere 219:191-201. https://doi.org/10.1016/j.chemo sphere.2018.12.007

Ribeiro AR, Castro PML, Tiritan ME (2012a) Chiral pharmaceuticals in the environment. Environ Chem Lett 10(3):239-253. https:// doi.org/10.1007/s10311-011-0352-0

Ribeiro AR, Castro PML, Tiritan ME (2012b) Environmental Fate of Chiral Pharmaceuticals: Determination, Degradation and Toxicity. In: Lichtfouse E, Schwarzbauer J, Robert D (eds) Environmental chemistry for a sustainable world: volume 2 : remediation of air and water pollution. Springer, Dordrecht, pp 3-45. https:// doi.org/10.1007/978-94-007-2439-6_1

Ribeiro AR, Afonso CM, Castro PML, Tiritan ME (2013) Enantioselective HPLC analysis and biodegradation of atenolol, metoprolol and fluoxetine. Environ Chem Lett 11:83-90. https://doi. org/10.1007/s10311-012-0383-1

Ribeiro AR, Santos LHMLM, Maia AS, Delerue-Matos C, Castro PML, Tiritan ME (2014) Enantiomeric fraction evaluation of pharmaceuticals in environmental matrices by liquid chromatography-tandem mass spectrometry. J Chromatogr A 1363:226235. https://doi.org/10.1016/j.chroma.2014.06.099

Riviere JE, Papich MG (eds) (2009) Veterinary pharmacology and therapeutics, 9th edn. Wiley-Blackwell, Hoboken, p 471

Roberts PH, Thomas KV (2006) The occurrence of selected pharmaceuticals in wastewater effluent and surface waters of the lower Tyne catchment. Sci Total Environ 356(1):143-153. https://doi. org/10.1016/j.scitotenv.2005.04.031

Rudy AC, Liu Y, Brater DC, Hall SD (1998) Stereoselective pharmacokinetics and inversion of (R)- ketoprofen in healthy volunteers. J Clin Pharmacol 38(2S):3S-10S. https://doi. org/10.1002/j.1552-4604.1998.tb04411.x

Sanganyado E, Lu Z, Fu Q, Schlenk D, Gan J (2017) Chiral pharmaceuticals: a review on their environmental occurrence and fate processes. Water Res 124:527-542. https://doi.org/10.1016/j. watres.2017.08.003

Sanganyado E, Lu Z, Liu W (2020) Application of enantiomeric fractions in environmental forensics: Uncertainties and inconsistencies. Environ Res 184:109354. https://doi.org/10.1016/j.envre s.2020.109354

Scriba GKE (2016) Chiral recognition in separation science-an update. J Chromatogr A 1467:56-78. https://doi.org/10.1016/j. chroma.2016.05.061

Sugawara Y, Fujihara M, Miura Y, Hayashida K, Takahashi T (1978) Studies on the fate of naproxen II. Metabolic fate in various animals and man. Chem Pharm Bull 26(11):3312-3321. https://doi. org/10.1248/cpb.26.3312

Suzuki T, Kosugi Y, Hosaka M, Nishimura T, Nakae D (2014) Occurrence and behavior of the chiral anti-inflammatory drug naproxen in an aquatic environment. Environ Toxicol Chem 33(12):26712678. https://doi.org/10.1002/etc.2741

Svan A, Hedeland M, Arvidsson T, Jasper JT, Sedlak DL, Pettersson CE (2015) Rapid chiral separation of atenolol, metoprolol, propranolol and the zwitterionic metoprolol acid using supercritical fluid chromatography-tandem mass spectrometry-application to wetland microcosms. J Chromatogr A 1409:251-258. https:// doi.org/10.1016/j.chroma.2015.07.075

Thomason MJ, Hung YF, Rhys-Williams W, Hanlon GW, Lloyd AW (1997) Indirect enantiomeric separation of 2-arylpropionic acids and structurally related compounds by reversed phase HPLC. J 
Pharm Biomed Anal 15(11):1765-1774. https://doi.org/10.1016/ S0731-7085(96)01979-6

Tijani JO, Fatoba OO, Babajide OO, Petrik LF (2016) (2016) Pharmaceuticals, endocrine disruptors, personal care products, nanomaterials and perfluorinated pollutants: a review. Environ Chem Lett 14:27-49. https://doi.org/10.1007/s10311-015-0537-z

Tiritan ME, Fernandes C, Maia AS, Pinto M, Cass QB (2018) Enantiomeric ratios: Why so many notations? J Chromatogr A 1569:1-7. https://doi.org/10.1016/j.chroma.2018.07.039

Upton RA, Buskin JN, Williams RL, Holford NHG, Riegelman S (1980) Negligible excretion of unchanged ketoprofen, naproxen, and probenecid in urine. J Pharm Sci 69(11):1254-1257. https:// doi.org/10.1002/jps.2600691105

Verlicchi P, Al Aukidy M, Zambello E (2012) Occurrence of pharmaceutical compounds in urban wastewater: Removal, mass load and environmental risk after a secondary treatment-a review. Sci Total Environ 429:123-155. https://doi.org/10.1016/j.scito tenv.2012.04.028

Villanueva M, Heckenberger R, Strobach H, Palmer M, Schror K (1993) Equipotent inhibition by $\mathrm{R}(-)-, \mathrm{S}(+)$ - and racemic ibuprofen of human polymorphonuclear cell function in vitro. Br J Clin Pharmacol 35(3):235-242. https://doi.org/10.1111/j.1365-2125.1993. tb05690.x

Wang F, Wang B, Qu H, Zhao W, Duan L, Zhang Y, Zhou Y, Yu G (2020) The influence of nanoplastics on the toxic effects, bioaccumulation, biodegradation and enantioselectivity of ibuprofen in freshwater algae Chlorella pyrenoidosa. Environ Pollut 263:114593. https://doi.org/10.1016/j.envpol.2020.114593

Wang Z, Zhao P, Zhu B, Jiang Z, Guo X (2018) Magnetic solid-phase extraction based on $\mathrm{Fe}_{3} \mathrm{O}_{4}$ /graphene nanocomposites for enantioselective determination of representative profens in the environmental water samples and molecular docking study on adsorption mechanism of graphene. J Pharm Biomed Anal 156:88-96. https ://doi.org/10.1016/j.jpba.2018.04.023

Welch CJ (1994) Evolution of chiral stationary phase design in the Pirkle laboratories. J Chromatogr A 666(1):3-26. https://doi. org/10.1016/0021-9673(94)80367-6
Winkler M, Lawrence JR, Neu TR (2001) Selective degradation of ibuprofen and clofibric acid in two model river biofilm systems. Water Res 35(13):3197-3205. https://doi.org/10.1016/S0043 -1354(01)00026-4

Wong CS (2006) Environmental fate processes and biochemical transformations of chiral emerging organic pollutants. Anal Bioanal Chem 386(3):544-558. https://doi.org/10.1007/s0021 6-006-0424-3

Wsol V, Skalova L, Szotakova B (2004) Chiral inversion of drugs: coincidence or principle? Curr Drug Metab 5(6):517-533. https ://doi.org/10.2174/1389200043335360

Yuan X, Li X, Guo P, Xiong Z, Zhao L (2018) Simultaneous enantiomeric analysis of chiral non-steroidal anti-inflammatory drugs in water, river sediment, and sludge using chiral liquid chromatography-tandem mass spectrometry. Anal Methods 10(36):44044413. https://doi.org/10.1039/C8AY01417E

Zha D, Li Y, Wang L, Yang C, Lu G (2017) Occurrence and attenuation of pharmaceuticals and their transformation products in rivers impacted by sewage treatment plants. RSC Adv 7(65):4090540913. https://doi.org/10.1039/C7RA06852B

Zhang W, Song Y, Chai T, Liao G, Zhang L, Jia Q, Qian Y, Qiu J (2020) Lipidomics perturbations in the brain of adult zebrafish (Danio rerio) after exposure to chiral ibuprofen. Sci Total Environ 713:136565. https://doi.org/10.1016/j.scitotenv.2020.136565

Zojaji P, Alhachami H, Kariminezhad E, Jauffur S, Bakhshi Z, Vaudreuil MA, Sauve S, Elektorowicz M (2019) Occurrence of ibuprofen and 2-hydroxy ibuprofen in Saint Lawrence river. In: Proceedings, annual conference-Canadian society for civil engineering

Zuloaga O, Navarro P, Bizkarguenaga E, Iparraguirre A, Vallejo A, Olivares M, Prieto A (2012) Overview of extraction, clean-up and detection techniques for the determination of organic pollutants in sewage sludge: a review. Anal Chim Acta 736:7-29. https://doi.org/10.1016/j.aca.2012.05.016

Publisher's Note Springer Nature remains neutral with regard to jurisdictional claims in published maps and institutional affiliations. 